\title{
Aspectos ecológicos de briofitas em areas preservadas de mata atlantica, Rio Janeiro, Brasil
}

\author{
M. Isabel M. N. de Oliveira-e-Silva. ${ }^{1}$ \\ Adauto Ivo Milanez ${ }^{2}$ \\ Olga Yano ${ }^{2}$
}

1. Universidade do Estado do Rio de Janeiro, Rua São Francisco Xavier 524, IBRAG, DBAV, 20550-013 Rio de Janeiro, RJ, Brasil - e-mail: mabelmatos@hotmail.com

2. Instituto de Botânica, Caixa Postal 4005, 01061-970 São Paulo, SP, Brasil.

\begin{abstract}
This paper aims at comparing the continental (Mangaratiba) and insular (Ilha Grande) bryoflora in the state of Rio de Janeiro. The floristic survey of these areas has resulted in the identification of 231 bryophyte species, the similarity index being $69 \%$. The highest index of specific diversity occurs in a track that is not under the influence of local human activity. The bryoflora is represented by families typical of tropical rain forests such as Lejeuneaceae, which also presents the highest index of specific diversity. The majority of the species presents rare absolute frequency, and is saxicolous, umbraticolous and ombrophilous. Nevertheless, these characteristics are not limiting as morphological adaptations allow for the colonization of different environments and substrates.
\end{abstract}

Resumo: Neste trabalho são comparadas as briofloras continental (Mangaratiba) e insular (Ilha Grande) de Mata Atlântica do estado do Rio de Janeiro. O inventário florístico dessas áreas resultou na identificação de 231 espécies de briófitas, sendo 69\% o índice de similaridade entre elas. O maior índice de diversidade específica ocorre em uma trilha que não sofre influência das atividades antrópicas locais. A brioflora estudada está representada por famílias típicas das florestas tropicais úmidas como por exemplo, Lejeuneaceae, que também apresenta a maior riqueza específica. A maioria das espécies, apresenta freqüência absoluta muito rara e é saxícola, umbrófila e ombrófila, embora estas características não sejam limitantes, pois adaptações morfológicas permitem a colonização de ambientes e substratos diferentes. 


\section{Introdução}

Poucos são os trabalhos sobre a ecologia das briófitas tanto no Brasil como no mundo. Daqueles realizados por pesquisadores estrangeiros podem ser citados os de Barkman (1958) que estudou a fitossociologia e ecologia das criptógamas epífitas; Proctor (1979) que correlacionou a estrutura e as adaptações ecofisiológicas; Richards (1984); Gradstein (1992); Gradstein \& Pócs (1989) e Frahm \& Gradstein (1991) que estudaram a ecologia das briófitas das florestas tropicais. No Brasil, podem ser relacionados os trabalhos de Lisboa (1976) sobre a brioecologia de uma campina amazônica; Pôrto (1992) comparando duas florestas do estado de Pernambuco e Almeida Sá (1995) que apresentou os aspectos ecológicos das briófitas próximas do Riacho Coité também no estado de Pernambuco. Segundo Koponen (1978) os dados ecológicos em briologia são de grande ajuda na identificação de espécies.

O inventário da brioflora das áreas estudadas, possibilitou o conhecimento de novos táxons para o Brasil (Oliveira-e-Silva \& Yano 1998) e permitiu a análise de alguns aspectos ecológicos, como os diferentes tipos de substratos e de ambientes colonizados pelas briófitas, a diversidade específica das famílias encontradas e das trilhas percorridas, a freqüência absoluta das espécies, o índice de similaridade entre as áreas estudadas nos dois municípios e essas áreas com a de outras localidades.

O objetivo deste trabalho é comparar a brioflora continental da Reserva Ecológica de Rio das Pedras - RERP, com a insular da Ilha Grande (PEIG e RBPS) e fornecer dados sobre a ecologia das briófitas de Mata Atlântica, ecossistema em alto processo de desmatamento.

\section{Material e métodos}

A Reserva Ecológica de Rio das Pedras (RERP), está situada na altura do km 54 da Rodovia RioSantos BR-101, município de Mangaratiba, estado do Rio de Janeiro, entre as coordenadas $22^{\circ} 59^{\prime} \mathrm{S}-44^{\circ} 05^{\prime} \mathrm{W}$ (Fig. 1). É recortada por numerosos rios alimentados por diminutos regatos, que compondo a Bacia do Rio Grande que nasce, corre e deságua em seus domínios. Apresenta altitudes entre 20-1150m que devido a forte interferência antrópica só apresenta mata primária a partir dos $420 \mathrm{~m}$ de altitude.

A Ilha Grande situada no município de Angra dos Reis, estado do Rio de Janeiro, entre as coordenadas $23^{\circ} \mathrm{S}-44^{\circ} \mathrm{W}$ (Fig. 1), tem $155 \mathrm{~km}$ de litoral e $193 \mathrm{~km}^{2}$ de área onde estão distribuídas vilas, o Parque Estadual da Ilha Grande (PEIG) com $56 \mathrm{~km}^{2}$ e a Reserva Biológica Estadual da Praia do Sul (RBEPS) com $36 \mathrm{~km}^{2}$.

As áreas estudadas estão inseridas na zona de domínio ecológico da Mata Atlântica, onde predomina a Floresta Ombrófila Densa (de terras baixas, submontana e montana) e em menor proporção as áreas das formações pioneiras de influência marinha (restinga) e fluviomarinhas (manguezal). Apresentam clima ombrófilo, sem déficit hídrico e com acentuada influência marinha. A temperatura média anual é de $22,5^{\circ} \mathrm{C}$, sendo fevereiro o mês mais quente, $\operatorname{com} 25,7^{\circ} \mathrm{C}$ e o mais frio julho, com $19,6^{\circ} \mathrm{C}$ (Estação Meteorológica de Angra dos Reis - INMET).

Nas duas áreas as coletas foram aleatórias. $\mathrm{Na}$ RERP ocorreram com a periodicidade de quinze dias nos três primeiros semestres e mensalmente no último semestre, no período de outubro de 1992 até outubro de 1994, em um total de 31 expedições e 23 trilhas percorridas. Na Ilha Grande foram mensais, no período de junho de 1994 até maio de 1995, em um total de 20 expedições e 17 trilhas .

Foram analisados: o coeficiente de similaridade entre a brioflora continental e a insular, o coeficiente de similaridade entre essas duas áreas e áreas do município de Nova Friburgo (Costa 1992 e 1994) e de Itatiaia (Dusén 1903 e 1909) no estado do Rio de Janeiro e áreas do estado do Espírito Santo (Schäfer-Verwimp 1991); a diversidade específica de cada uma das trilhas percorridas na RERP e na Ilha Grande, a diversidade específica das duas áreas como um todo; a freqüência absoluta das espécies; os ambientes e substratos preferenciais.

Para determinar o grau de similaridade foi usado o coeficiente de similaridade de Sorensen (S) onde o número de espécies comuns as duas áreas (a) é comparado com o número total das espécies em cada uma das duas áreas (b e c), usando a formula $S=2 a / 2 a+b+c$ (Valentin 1995). 
Para calcular a diversidade específica (Hs) de cada trilha, foi utilizada a fórmula $\mathrm{Hs}=-\mathrm{E}$ Pi Ln Pi (Alcayaga 1985), onde: Pi - abundância relativa de cada espécie na trilha e Ln Pi - o logaritmo neperiano de Pi.

Para calcular a freqüência absoluta das espécies foi usada a fórmula $F=n 100 / N$, onde: $F$ freqüência da espécie $\mathrm{X} ; \mathrm{n}$ - número de trilhas onde a espécie $\mathrm{X}$ foi registrada e $\mathrm{N}$ - número total de trilhas (Dajoz 1983). O número de classes de freqüência $(\mathrm{CF})$ foi determinado segundo Dajoz (1983), com algumas modificações, estabelecendo-se seis classes, a saber: muito abundantes, abundantes, freqüentes, pouco freqüentes, raras e muito raras.

O calculo da amplitude de classe (A) foi feito segundo Alcayaga (1985) onde $A=<$ freqüência absoluta - > freqüência absoluta/ $\mathrm{n}^{\circ} \mathrm{CF}$, ajustandose os valores em suas casas decimais.

\section{Resultados e discussão}

\section{1 - Índice de similaridade}

Foram identificadas 231 espécies nas áreas estudadas (Tab. 1). Considerando-se outros inventários florísticos realizados em áreas mais extensas e por períodos maiores, como o de Sim (1926) que relacionou 666 espécies para a África do Sul, Sehnem (1969-1980) que listou 430 táxons de musgos para os estados do Paraná, Rio Grande do Sul e Santa Catarina e SchäferVerwimp (1991) que citou 295 espécies de briófitas para o estado do Espírito Santo, o número de espécies identificadas para as áreas de Mata Atlântica do Rio de Janeiro, pode ser considerado alto.

O número de espécies comuns às duas áreas é de 120 (Tab. 1). Dezoito táxons foram coletados exclusivamente na RERP e 93 na Ilha Grande, levando a um índice de similaridade de $69 \%$. Considerando-se as duas áreas como um todo e outras áreas de Mata Atlântica, os coeficientes encontrados são os seguintes: $38 \%$ comparandose com a brioflora do estado do Espírito Santo (Schäfer- Verwimp 1991); 31\% com Nova Friburgo (Costa 1992 e 1994) e 9,45\% com a Serra do Itatiaia (Dusén 1903 e 1909), estado do Rio de Janeiro (Fig. 2).

$\mathrm{O}$ índice de similaridade entre a RERP e a Ilha Grande (Fig. 2) mostra que os dois ambientes mantiveram alguma ligação no passado e que as diferenças específicas existentes ocorrem pelas modificações ambientais causadas pelo homem. Na Ilha Grande há muitas áreas de mata primária enquanto que na RERP o ambiente encontra-se em pleno processo de regeneração. A similaridade entre essas duas áreas e outras áreas de Mata Atlântica do estado do Rio de Janeiro, como Nova Friburgo e Itatiaia e do estado do Espírito Santo, é baixa (Fig. 2) demonstra que regiões diversas de um mesmo ecossistema possuem riqueza específica própria. É importante ressaltar que o menor índice ocorre entre as duas áreas e a Serra do Itatiaia que apresenta altitudes superiores às da RERP e da Ilha Grande.

Considerando-se o inventário florístico de outras ilhas no território brasileiro e o da Ilha Grande, foram encontradas apenas 14 espécies de briófitas ocorrendo igualmente na Ilha de Marajó (Lisboa \& Maciel 1994), oito na Ilha de Maracá (Milliken \& Ratter 1989 e Yano 1992a), seis na Ilha de Fernando de Noronha (Vital et al. 1991) e quatro na Ilha do Cardoso (Yano 1990), o que pode ser explicado pelo pequeno número de espécies inventariadas em cada uma destas ilhas, 32 na Ilha de Marajó, 23 na Ilha de Maracá, 20 na Ilha de Fernando de Noronha e oito na Ilha do Cardoso.

\section{Diversidade específica}

O coeficiente de Shannon revelou maior diversidade específica (Hs) na Ilha Grande $(4,7346)$ do que na RERP $(2,6908)$ (Fig. 3).

Dentre as trilhas percorridas, a do Pico do Papagaio (Tab. 2) na Ilha Grande apresenta o maior índice de diversidade $(\mathrm{Hs}=4,3976)$ seguida da trilha Abraão - Vila Dois Rios (Hs = 4,3421) e as trilhas T 22 e T 1 na RERP (Tab. 2) apresentam o menor índice $(\mathrm{Hs}=0$ e Hs $=1,0836$, respectivamente). Slack (1977) comenta que entre áreas de tamanhos diferentes, a maior não é necessariamente a de maior diversidade específica, entretanto áreas com altitudes diferentes, a mais elevada apresenta o maior índice de diversidade. A Ilha Grande apresenta um índice de diversidade específica superior à da RERP (Fig. 3) e apresenta também as maiores altitudes (Tab. 2). Dentre as trilhas o maior índice de diversidade encontrado está no Pico do Papagaio na Ilha Grande (Tab. 2) onde ocorrem 
Tab. 1: Freqüência Absoluta (F. A.) e Classes de Freqüência (C.F.: Muito Abundantes = MA; Abundantes $=$ AB; Frequiente $=$ F; Pouco Freqüente $=$ PF; Rara $=$ R; Muito Rara $=$ MR; Ausente $=\mathrm{AU}$ ) das espécies coletadas nas áreas de estudo (continua).

Famílias e Espécies coletadas $\begin{array}{ll}\text { F. A } \% & \text { C.F. } \\ \text { RERP } & \end{array}$ RERP

ANTHOCEROTACEAE

Anthoceros punctatus L.

Phaeoceros laevis (L.) Prosk.

HERBERTACEAE

Herbertus angustivittatus (Steph.) Fulf.

$H$. divergens (Steph.) Herz.

TRICHOCOLEACEAE

Trichocolea flaccida (Spruce) Spruce LEPIDOZIACEAE

Arachniopsis diacantha (Mont.) Howe

Bazzania pallide-virens (Steph.) Fulf.

B. stolonifera (Sw.) Trev.

Kurzia brasiliensis (Steph.) Grolle

$K$. capillaris (Sw.) Grolle var. verrucosa

(Steph.) Pócs

Lepidozia inaequalis (Lehm. \& Lindenb.)

Gott. et al.

Telaranea nematodes (Gott. ex. Aust.) Howe

CALYPOGEIACEAE

Calypogeia miquelli Mont.

CEPHALOZIELLACEAE

Kymatocalyx dominicensis (Spruce) Váña GEOCALYCACEAE

Lophocolea bidentata (L.) Dum.

L. martiana Nees

PLAGIOCHILACEAE

Plagiochila distinctifolia Lindenb.

P. kroneana Steph.

$P$. martiana (Nees) Lindenb.

$P$. montagnei Nees

$P$. rutilans Lindenb.

P. scissifolia Steph.

$P$. subplana Lindenb.

Plagiochila sp 1.

Plagiochila sp 2.

BALANTIOPSIDACEAE

Isotachis aubertii (Schwaegr.) Mitt.

RADULACEAE

Radula javanica Gott.

R. marginata (Tayl.) Mitt.

R. mexicana Lindenb. \& Gott.

PORELLACEAE

Porella brasiliensis (Raddi) Schiffn.

FRULLANIACEAE

Frullania arecae (Spreng.) Gott.

$F$. beyrichiana (Lehm. \& Lindenb.) Lehm. \&

Lindenb.

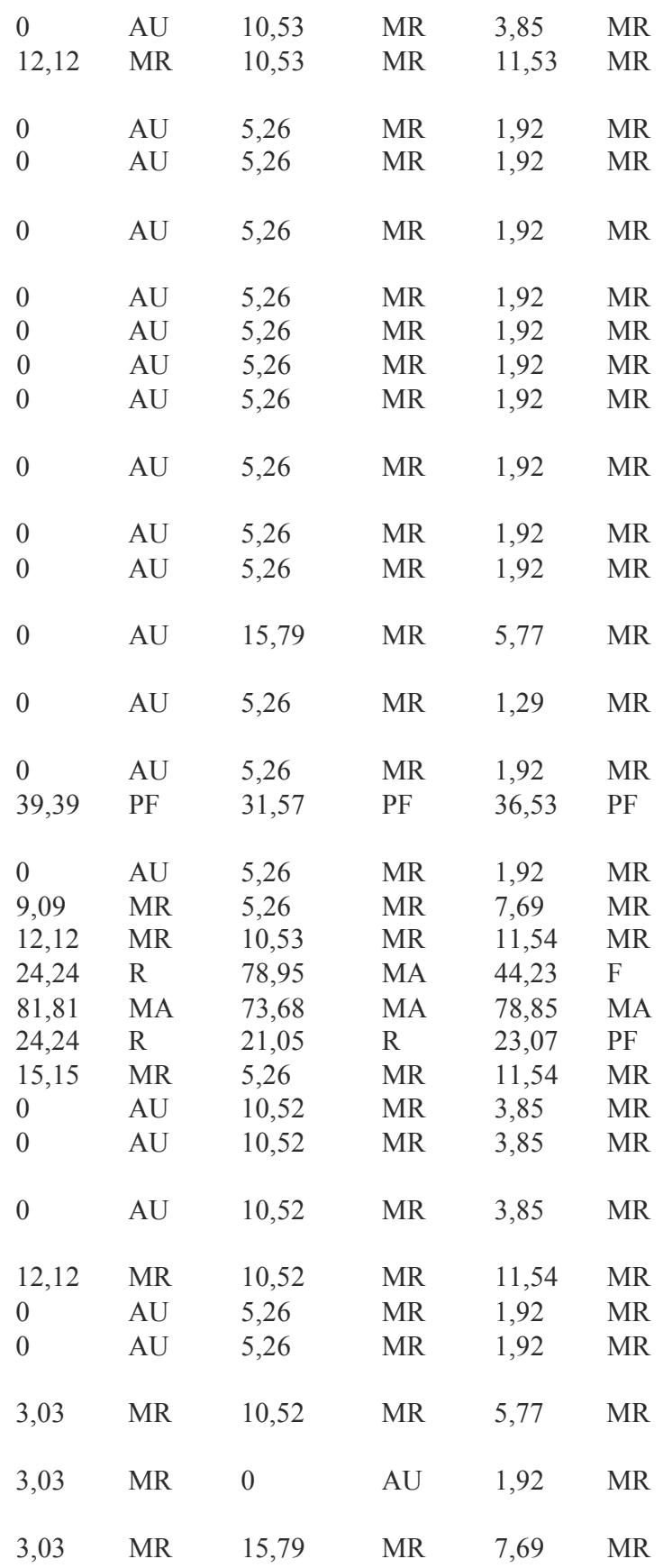

TROPICAL BRYOLOGY 22 (2002) 
F. brasiliensis Raddi

$F$. caulisequa (Nees) Nees

F. dilatata (L.) Dum.

$F$. ericoides (Nees) Nees

F. montagnei Gott.

$F$. neesii Lindenb.

F. riojaneirensis (Raddi) Aongstr.

$F$. setigera Steph.

F. supradecomposita (Lehm. \& Lindenb.)

Lehm. \& Lindenb.

LEJEUNEACEAE.

Acrolejeunea emergens (Mitt.) Steph.

Aphanolejeunea diaphana (Evans) Schust.

Archilejeunea parviflora (Nees) Schiffn.

Bryopteris diffusa (Sw.) Nees

B. filicina (Sw.) Nees

Caudalejeunea lehmanniana (Gott.) Steph.

Ceratolejeunea cubensis (Mont.) Schiffn.

C. rubiginosa Steph.

Cheilolejeunea rigidula (Nees ex Mont.) Schust

C. trifaria (Reinw. et al.) Mizut.

Cololejeunea obliqua Nees \& Mont.

Colura tortifolia (Mont. ex Nees) Trev.

Diplasiolejeunea brunnea Steph.

D. pellucida (Meiss.) Schiffn.

Drepanolejeunea mosenii (Steph.) Bischler

D. orthophylla (Nees \& Mont.) Bischler

LEJEUNEACEAE.

Harpalejeunea uncinata Steph.

Lejeunea bermudiana (Evans) Schust.

L. caespitosa Lindenb. ex G.L. \& Nees

L. cancellata Nees \& Mont.

L. flava (Sw.) Nees

L. glaucescens Gott.

L. laete-virens Nees \& Mont. ex Mont.

L. minutiloba Evans

L. pterogonia (Lehm. \& Lindenb.) Mont.

Leptolejeunea elliptica (Lehm. \& Lindenb.)

Schiffn.

L. moniliata Steph.

Leucolejeunea unciloba (Lindenb.) Evans

L. xanthocarpa (Lehm. \& Lindenb.) Evans

Lopholejeunea nigricans (Lindenb.) Schiffn.

L. subfusca (Nees) Steph.

Marchesinia brachiata (Sw.) Schiffn. $\quad 3,03$

Omphalanthus filiformis (Sw.) Nees

Rectolejeunea phyllobola (Nees \& Mont.) Evans 0

$R$. pililoba (Spruce) Schust.

Stictolejeunea squamata (Willd. ex Web.) Schiffn.0

Symbiezidium transversale (Sw.) Trev.

FOSSOMBRONIACEAE

Fossombronia foveolata S. O. Lindb.

PALLAVICINIACEAE

Symphyogyna aspera Steph. ex McCormick

3,03

0

3,03

12,12

0

0

0

0

0

3,03

6,06

3,03

3,03

3,03

3,03

81,81

45,45

33,33

6,06

24,24

$\begin{array}{lll}18,18 & \mathrm{R} & 52,63 \\ 3,03 & \mathrm{MR} & 5,26 \\ 0 & \mathrm{AU} & 5,26 \\ 27,22 & \mathrm{R} & 47,37 \\ 0 & \mathrm{AU} & 5,26 \\ 3,03 & \mathrm{MR} & 10,52 \\ 12,12 & \mathrm{MR} & 10,52 \\ 0 & \text { AU } & 5,26 \\ & & \\ 6,06 & \mathrm{MR} & 26,32\end{array}$

F

30,77

MR

MR $\quad 1,92$

PF

$1,92 \quad \mathrm{MR}$

F 34,61 PF

MR $1,92 \quad$ MR

MR 5,77 MR

MR $11,54 \quad$ MR

MR $1,92 \quad$ MR

$\begin{array}{llll}M R & 26,32 & R & 13,46\end{array}$

$0 \quad$ AU $\quad 10,52$

12,12 MR

$0 \quad$ AU

3,03 MR

$0 \quad$ AU

MR

AU

MR

MR

MR

AU

AU

AU

AU

AU

0

5,26

5,26

36,84

5,26

21,05

31,57

15,78

52,63

5,26

5,26

5,25

5,26

5,26

MR

10,23

MR $\quad 3,85$

AU $\quad 7,69$

MR $\quad 1,92$

MR $\quad 3,85$

PF $\quad 40,98$

MR $\quad 7,69$

R $\quad 7,69$

PF $\quad 13,46$

MR $\quad 7,69$

F 26,92

MR 1,92

MR $\quad 1,92$

MR $\quad 1,92$

MR $\quad 1,92$

MR 1,92

MR $\quad 5,77$

MR $\quad 5,77$

5,26

AU

1,92

3,85

MR

AU

1,92

$\begin{array}{ll}\text { AB } & 55,77 \\ \text { MA } & 76,92\end{array}$

PF $\quad 42,31$

R 28,85

MR $\quad 5,77$

PF 21,05

MR $\quad 5,26$

$0 \quad$ AU $\quad 5,26$

3,03 MR 10,53

MR $\quad 1,92$

MR $\quad 5,77$

MR 1,92

MR 3,85

MR $\quad 3,85$

PF 28,85

AB 23,08

MR $\quad 1,92$

MR 1,922

MR $\quad 3,85$

MR $\quad 5,77$

MR 3,85

MR $\quad 1,92$

MR $1,92 \quad$ MR

$0 \quad \mathrm{AU} \quad 5,26$

R

42,10

PF

30,77

PF 
S. brasiliensis Nees \& Mont. ANEURACEAE

Riccardia alata (Steph.) Hell

R. chamedryfolia (With.) Grolle

$R$. metzgeriiformis (Steph.) Hell

R. regnellii (Aongstr.) Hell

METZGERIACEAE

Metzgeria albinea Spruce

$M$. angusta Steph.

M. aurantiaca Steph.

M. convoluta Steph.

M. dichotoma (Sw.) Nees

M. furcata (L.) Corda

MONOCLEACEAE

Monoclea gottschei Lindb. ssp. elongata

Gradst. \& Mues

WIESNERELLACEAE

Dumortiera hirsuta (Sw.) Nees

MARCHANTIACEAE

Marchantia berteroana Lehm. \& Lindenb.

M. chenopoda L.

M. papillata Raddi

POLYTRICHACEAE

Pogonatum pensilvanicum (Hedw.) P. Beauv.

Polytrichum brasiliense Hampe

P. commune Hedw.

$\begin{array}{llllll}6,06 & \text { MR } & 36,84 & \text { PF } & 17,31 & \mathrm{R}\end{array}$

$\begin{array}{llllll}0 & \text { AU } & 5,26 & \text { MR } & 1,92 & \text { MR }\end{array}$

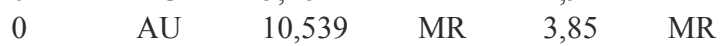

3,03 MR 31,58 PF $13,46 \quad$ MR

$\begin{array}{lllll}0 & \text { AU } & 15,79 & \text { MR } & 5,77\end{array}$

$\begin{array}{llllll}9,09 & \text { MR } & 10,53 & \text { MR } & 9,62 & \text { MR }\end{array}$

$\begin{array}{llllll}27,27 & \mathrm{R} & 5,26 & \mathrm{MR} & 19,23 & \mathrm{R}\end{array}$

$\begin{array}{llllll}3,03 & \text { MR } & 10,53 & \text { MR } & 13,46 & \text { MR }\end{array}$

$\begin{array}{llllll}18,18 & \mathrm{R} & 15,79 & \mathrm{MR} & 17,31 & \mathrm{R}\end{array}$

15,15 MR $0 \quad$ AU $9,62 \quad$ MR

$\begin{array}{lllll}24,24 & R & 0 & \text { AU } & 15,38\end{array}$

$\begin{array}{llllll}30,30 & \text { PF } & 31,58 & \text { PF } & 30,77 & \text { PF }\end{array}$

45,45 F 26,32

$\mathrm{R}$

$0 \quad$ AU

5,26

3,03

0

MR

15,79

MR

38,46

PF

$\mathrm{AU}$

10,53

MR

1,92

MR

$0 \quad$ AU

10,53

MR

7,69

MR

$0 \quad$ AU

5,26

MR

3,85

MR

AU

5,26

MR

1,92

MR

MR

1,92

MR

$P$. juniperinum Hedw.

\section{ORTHOTRICHACEAE}

Groutiella tomentosa (Hornsch.) Wijk \& Marg.

G. tumidula (Mitt.) Vitt

Macrocoma frigidum (C. Muell.) Vitt

Macromitrium argutum Hampe

M. microstomum (Hook. \& Grev.) Schwaegr.

M. pellucidum Mitt.

M. punctatum (Hook. \& Grev.) Brid.

M. richardii Schwaegr.

ORTHOTRICHACEAE

Schlotheimia jamesonii (Arnott) Brid.

S. rugifolia (Hook.) Schwaegr.

(1)

MR $\quad 10,53$

$\begin{array}{lll}M R & 5,77\end{array}$

ERPODIACEAE

Erpodium glaziouii Hampe

HELICOPHYLLACEAE

Helicophyllum torquatum (Hook.) Brid.

\section{0}

AU

AU

15,79

MR

MR

15,53

3,03 MR

MR

0

AU

15,79

5,26

42,10

3.03

6,06

MR

15,79

15,79

$0 \quad \mathrm{AU}$

21,05

3,03

MR

26,31

3,03

MR

0

12,12 MR

15,79

$0 \quad \mathrm{AU}$

21,05

Brachymenium systylium (C. Muell.) Jaeg.

Bryum argenteum Hedw.

0

B. billardieri Schwaegr.

$B$. densifolium Brid.

3,03

6,06

B. limbatum C. Muell.

B. matto-grossense Broth.

B. pabstianum C. Muell.

B. paradoxum Schwaegr.

B. renauldii Röl ex Ren. \& Card.

AU

5,26

5,26

36,84

3,03

0

0

0

MR

$\mathrm{MR}$

MR

0

10,53

5,26

AU

31,58

AU

5,26

Plagiomnium rhynchophorum (Hook. f.) T. Kop. 15,15

MR
0 $\begin{array}{lll}\text { MR } & 5,77 \quad \text { MR }\end{array}$

MR 3,85 MR

MR $1,92 \quad$ MR

PF 21,15 R

MR 7,69 MR

MR $9,61 \quad$ MR

$\begin{array}{lll}\mathrm{R} & 7,69 & \mathrm{MR}\end{array}$

R $11,54 \quad$ MR

MR $13,46 \quad$ MR

$\begin{array}{lll}\mathrm{R} & 7,69 & \mathrm{MR}\end{array}$

$\begin{array}{lll}\text { MR } & 1.92 \quad \text { MR }\end{array}$

MR 3,85 MR

PF 17,31 R

AU $1,92 \quad$ MR

MR $3,85 \quad$ MR

MR $3,85 \quad$ MR

PF 28,85 PF

MR $1,92 \quad$ MR

AU $\quad 9,62 \quad$ MR
AU $\quad 1,92 \quad$ MR 


\section{MNIACEAE}

Pyrrhobryum spiniforme (Hedw.) Mitt. RHIZOGONIACEAE

Hymenodon aeruginosus (Hook. f. \& Wils.)

C. Muell.

BARTRAMIACEAE

Philonotis hastata (Duby) Wijk \& Marg.

P. uncinata (Schwaegr.) Brid.

THUIDIACEAE

Haplocladium microphyllum (Hedw.) Broth.

Thuidium tomentosum Besch.

RACOPILACEAE

Racopilum tomentosum (Hedw.) Brid.

MYRINIACEAE

Helicodontium capillare (Hedw.) Jaeg.

\section{BRACHYTHECIACEAE}

Aerolindigia capillacea (Hornsch.) Menzel

Steerecleus scariosus (Tayl.) Robins.

STEREOPHYLLACEAE

Eulacophyllum cultelliforme (Sull.)

Buck \& Ireland

Pilosium chlorophyllum (Hornsch.) C. Muell.

Stereophyllum radiculosum (Hook.) Mitt.

\section{HYPNACEAE}

Isopterygium tenerifolium Mitt.

Mittenothamnium elegantulum (Hook.) Card.

Vesicularia vesicularis (Schwaegr.) Broth.

ENTODONTACEAE.

Entodon beyrichii (Schwaegr.) C. Mue

Erythrodontium longisetum (Hook.) Par.

SEMATOPHYLLACEAE

Acroporium longirostre (Brid.) Buck

Sematophyllum adnatum (Michx.) Britt.

S. demissum (Wils.) Mitt.

$\begin{array}{llllll}15,15 & \text { MR } & 0 & \text { AU } & 9,62 & \text { R } \\ & & & & & \\ 3,03 & \text { MR } & 5,26 & \text { MR } & 3,85 & \text { MR } \\ & & & & & \\ 24,24 & \text { R } & 10,53 & \text { MR } & 19,23 & \text { R } \\ 9,09 & \text { R } & 26,32 & \text { R } & 15,38 & \text { R } \\ & & & & & \\ 3,03 & \text { MR } & 10,53 & \text { MR } & 5,77 & \text { MR } \\ 42,42 & \text { PF } & 47,37 & \text { F } & 5,77 & \text { MR } \\ & & & & & \\ 63,63 & \text { AB } & 26,32 & \text { R } & 50,00 & \text { F } \\ & & & & & \\ 78,78 & \text { MA } & 31,57 & \text { PF } & 61,54 & \text { AB } \\ & & & & & \\ 21,21 & \text { R } & 10,53 & \text { MR } & 17,30 & \text { R } \\ 69,69 & \text { AB } & 15,79 & \text { MR } & 50,00 & \text { F }\end{array}$

3,03 MR $\quad 10,53$

MR $5,77 \quad$ MR

12,12 MR 26,32

R $\quad 17,11 \quad \mathrm{R}$

$\begin{array}{llllll}0 & \mathrm{AU} & 5,26 & \mathrm{MR} & 1,92 & \mathrm{MR}\end{array}$

S. subpinnatum (Brid.) Britt.

S. subsimplex (Hedw.) Mitt.

Taxithelium planum (Brid.) Mitt.

Trichosteleum fluviale (Mitt.) Jaeg.

LESKEACEAE

Herpetineuron toccoae (Sull. \& Lesq.) Card.

FABRONIACEAE

Fabronia ciliaris (Brid.) Brid. var. wrightii

(Sull. ex Sull.) Buck

LEUCODONTACEAE

Pseudocryphaea domingensis (Spreng.) Buck

PTEROBRYACEAE

Calyptothecium duplicatum (Schwaegr.) Broth. 3,03

Jaegerina scariosa (Lor.) Arz.

Pireella cymbifolia (Sull.) Card.

75,75

MA

52,63

F

27,27 R $\quad 63,16$

67,31

84,84 MA

42,10

$\mathrm{AB} \quad 40,38$

PF $\quad 69,23$

$\mathrm{AB}$

$\begin{array}{llllll}3,03 & \text { MR } & 0 & \text { AU } & 1,92 & \text { MR }\end{array}$

$\begin{array}{lllll}3,03 & \text { MR } & 0 & \text { AU } & 1,92\end{array}$

$\begin{array}{llllll}6,06 & \text { MR } & 26,32 & \mathrm{R} & 13,46 & \mathrm{MR}\end{array}$

3,03 MR 10,52 $\quad$ MR $5,77 \quad$ MR

$\begin{array}{llllll}0 & \text { AU } & 5,26 & \text { MR } & 1,92 & \text { MR }\end{array}$

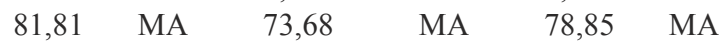

$\begin{array}{llllll}24,24 & R & 47,36 & F & 32,69 & \text { PF }\end{array}$

$\begin{array}{llllll}6,06 & \text { MR } & 26,32 & \mathrm{R} & 13,46 & \mathrm{MR}\end{array}$

$\begin{array}{llllll}6,06 & \text { MR } & 10,53 & \text { MR } & 7,69 & \text { MR }\end{array}$

$\begin{array}{llllll}3,03 & \text { MR } & 0 & \text { AU } & 1,92 & \text { MR }\end{array}$

Pterobryon densum (Schwaegr.) Hornsch.

METEORIACEAE

Floribundaria usneoides (Broth.) Broth.

Papillaria deppei (C. Muell.) Jaeg.

P. nigrescens (Hedw.) Jaeg.

Pilotrichella flexilis (Hedw.) Aongstr.

$\begin{array}{llllll}3,03 & \text { MR } & 5,26 & \text { MR } & 3,85 & \text { MR } \\ 0 & \text { AU } & 10,53 & \text { MR } & 3,85 & \text { MR } \\ & & & & & \\ 3,03 & \text { MR } & 0 & \text { AU } & 1,92 & \text { MR } \\ 0 & \text { AU } & 5,26 & \text { MR } & 11,92 & \text { MR } \\ 3,03 & \text { MR } & 0 & \text { AU } & 1,92 & \text { MR } \\ 0 & \text { AU } & 10,53 & \text { MR } & 3,85 & \text { MR } \\ & & & & & \\ 0 & \text { AU } & 10,53 & \text { MR } & 3,85 & \text { MR } \\ 3,03 & \text { MR } & 5,26 & \text { MR } & 3,85 & \text { MR } \\ 30,30 & \text { PF } & 26,51 & \text { R } & 28,85 & \text { PF } \\ 0 & \text { AU } & 10,53 & \text { MR } & 3,85 & \text { MR }\end{array}$


Orthostichella pentasticha (Brid.) Buck Squamidium leucotrichum (Tayl.) Broth. S. nigricans (Hook.) Broth.

Zelometeorium patulum (Hedw.) Manuel Z. recurvifolium (Hornsch.) Manuel NECKERACEAE

Neckeropsis disticha (Hedw.) Kindb.

N. undulata (Hedw.) Reichardt

$N$. villae-ricae (Besch.) Broth.

Porothamnium flagelliferum (C. Muell.) Fleisch.

Porotrichum substriatum (Hampe) Mitt.

PHYLLOGONIACEAE

Phyllogonium viride Brid.

PILOTRICHACEAE

Pilotrichum evanescens (C. Muell.) C. Muell. CALLICOSTACEAE

Callicostella merkelii (Hornsch.) Jaeg.

C. pallida (Hornsch.) Aongstr.

Crossomitrium patrisiae (Brid.) C. Muell.

Cyclodictyon varians (Sull.) O. Kuntze

Lepidopilum scabrisetum (Schwaegr.) Steere

Thamniopsis stenodictyon (Sehnem)

Oliveira-e-Silva \& O. Yano

T. incurva (Hornsch.) Buck

T. langsdorffii (Hook.) Buck

LEUCOMIACEAE

Leucomium strumosum (Hornsch.) Mitt.

HYPOPTERYGIACEAE

Hypopterygium tamarisci (Sw.) C. Muell.

Lopidium concinnum (Hook.) Wils.

POTTIACEAE

Barbula indica (Hook.) Spreng.

Chenia leptophylla (C. Muell.) Zand.

Hyophila involuta (Hook.) Jaeg.

Plaubelia sprengelii (Schwaegr.) Zand.

Tortella humilis (Hedw.) Jenn.

CALYMPERACEAE

Calymperes afzelii Sw.

C. erosum C. Muell.

C. lonchophyllum Schwaegr.

C. palisotii Schwaegr. ssp. richardii (C. Muell.)

S. Edwards

Syrrhopodon gaudichaudii Mont.

$S$. incompletus Schwaegr.

S. lycopodioides (Brid.) C. Muell.

$S$. parasiticus (Brid.) Besch.

S. prolifer Schwaegr. var. prolifer

S. prolifer var. papillosus (C. Muell.) Reese

S. rigidus Hook. \& Grev.

DICRANACEAE

Bryohumbertia filifolia (Hornsch.) J. P. Frahm Campylopus arctocarpus (Hornsch.) Mitt.

C. cryptopodioides Broth.

C. heterostachys (Hampe) Jaeg.

\begin{tabular}{|c|c|c|c|c|}
\hline 42,42 & $\mathrm{PF}$ & 47,37 & $\mathrm{~F}$ & 44,23 \\
\hline 9,09 & MR & 36,84 & PF & 19,23 \\
\hline 21,21 & $\mathrm{R}$ & 31,58 & $\mathrm{PF}$ & 25,00 \\
\hline 24,24 & $\mathrm{R}$ & 36,84 & PF & 28,85 \\
\hline 12,12 & MR & 10,53 & MR & 11,54 \\
\hline 81,81 & MA & 31,58 & $\mathrm{PF}$ & 63,46 \\
\hline 69,69 & $\mathrm{AB}$ & 57,89 & $\mathrm{AB}$ & 65,38 \\
\hline 9,09 & MR & 15,78 & MR & 11,54 \\
\hline 27,27 & $\mathrm{R}$ & 10,53 & MR & 21,15 \\
\hline 27,27 & $\mathrm{R}$ & 10,53 & MR & 21,15 \\
\hline 3,03 & MR & 21,05 & $\mathrm{R}$ & 11,54 \\
\hline 9,09 & MR & 0 & $\mathrm{AU}$ & 5,77 \\
\hline 18,18 & $\mathrm{R}$ & 36,84 & $\mathrm{PF}$ & 25,00 \\
\hline 39,39 & PF & 47,37 & $\mathrm{~F}$ & 42,30 \\
\hline 27,27 & $\mathrm{R}$ & 15,78 & MR & 23,07 \\
\hline 27,27 & $\mathrm{R}$ & 15,78 & MR & 23,07 \\
\hline 9,09 & MR & 21,05 & $\mathrm{R}$ & 13,46 \\
\hline 0 & $\mathrm{AU}$ & 5,26 & MR & 1,92 \\
\hline 30,30 & PF & 15,78 & MR & 25,00 \\
\hline 36,36 & PF & 15,78 & MR & 28,85 \\
\hline 12,12 & MR & 15,78 & MR & 13,46 \\
\hline 54,54 & $\mathrm{~F}$ & 47,37 & $\mathrm{~F}$ & 51,92 \\
\hline 0 & $\mathrm{AU}$ & 5,26 & MR & 1,92 \\
\hline 0 & $\mathrm{AU}$ & 5,26 & MR & 1,92 \\
\hline 0 & $\mathrm{AU}$ & 5,26 & MR & 1,92 \\
\hline 3,03 & MR & 42,10 & $\mathrm{PF}$ & 17,31 \\
\hline 0 & $\mathrm{AU}$ & 5,26 & MR & 1,92 \\
\hline 0 & $\mathrm{AU}$ & 21,05 & $\mathrm{R}$ & 7,69 \\
\hline 42,42 & PF & 57,89 & $\mathrm{AB}$ & 48,07 \\
\hline 36,36 & $\mathrm{PF}$ & 36,84 & $\mathrm{PF}$ & 36,54 \\
\hline 0 & $\mathrm{AU}$ & 5,26 & MR & 1,92 \\
\hline 9,09 & MR & 63,16 & $\mathrm{AB}$ & 28,85 \\
\hline 0 & $\mathrm{AU}$ & 5,26 & MR & 1,92 \\
\hline 21,21 & $\mathrm{R}$ & 36,84 & $\mathrm{PF}$ & 26,92 \\
\hline 0 & $\mathrm{AU}$ & 5,26 & MR & 1,92 \\
\hline 3,03 & MR & 0 & $\mathrm{AU}$ & 1,92 \\
\hline 6,06 & MR & 47,37 & $\mathrm{~F}$ & 21,15 \\
\hline 0 & $\mathrm{AU}$ & 21,05 & $\mathrm{R}$ & 7,69 \\
\hline 12,12 & MR & 10,53 & MR & 11,54 \\
\hline 6,06 & MR & 26,31 & $\mathrm{R}$ & 13,46 \\
\hline 0 & $\mathrm{AU}$ & 5,26 & MR & 1,92 \\
\hline 0 & $\mathrm{AU}$ & 10,53 & MR & 3,85 \\
\hline 12,12 & MR & 10,53 & MR & 11,54 \\
\hline
\end{tabular}




\section{DICRANACEAE}

Campylopus. pilifer Brid.

C. savannarum (C. Muell.) Mitt.

C. trachyblepharon (C. Muell.) Mitt.

Dicranella hilariana (Mont.) Mitt.

Holomitrium arboreum Mitt.

H. crispulum Mart.

H. olfersianum Hornsch.

Leucoloma cruegerianum (C. Muell.) Jaeg.

L. serrulatum Brid.

BRUCHIACEAE

Trematodon longicollis Michx.

LEUCOBRYACEAE

Leucobryum albicans (Schwaegr.) Lindb.

L. clavatum Hampe

L. crispum C. Muell.

L. martianum (Hornsch.) Hampe

Octoblepharum albidum Hedw.

O. cocuiense Mitt.

FISSIDENTACEAE

Fissidens asplenioides Hedw.

F. diplodus Mitt.

$F$. elegans Brid.

F. guianensis Mont.

$F$. intramarginatus (Hampe) Mitt.

$F$. longifalcatus C. Muell.

$F$. mollis Mitt.

F. prionodes Mont. fo. hornschuchii (Mont.)

Fleisch.

F. scariosus Mitt.

F. zollingeri Mont.

\begin{tabular}{|c|c|c|c|c|}
\hline 0 & AU & 21,05 & $\mathrm{R}$ & 7,69 \\
\hline 6,06 & MR & 26,31 & $\mathrm{R}$ & 13,46 \\
\hline 3,03 & MR & 21,05 & $\mathrm{R}$ & 9,61 \\
\hline 0 & $\mathrm{AU}$ & 5,26 & MR & 1,92 \\
\hline 0 & $\mathrm{AU}$ & 21,05 & $\mathrm{R}$ & 7,69 \\
\hline 0 & $\mathrm{AU}$ & 5,26 & MR & 1,92 \\
\hline 3,03 & MR & 5,26 & MR & 3,85 \\
\hline 0 & $\mathrm{AU}$ & 21,05 & $\mathrm{R}$ & 7,69 \\
\hline 3,03 & MR & 26,31 & $\mathrm{R}$ & 11,54 \\
\hline 6,06 & MR & 5,26 & MR & 5,77 \\
\hline 0 & $\mathrm{AU}$ & 5,26 & MR & 1,92 \\
\hline 0 & $\mathrm{AU}$ & 15,79 & MR & 5,77 \\
\hline 9,09 & MR & 15,79 & MR & 11,54 \\
\hline 6,06 & MR & 10,53 & MR & 7,69 \\
\hline 39,39 & $\mathrm{PF}$ & 79,95 & MA & 53,85 \\
\hline 9,09 & MR & 5,26 & MR & 7,69 \\
\hline 9,09 & MR & 42,10 & PF & 21,15 \\
\hline 18,18 & $\mathrm{R}$ & 31,58 & PF & 23,07 \\
\hline 0 & $\mathrm{AU}$ & 5,26 & MR & 1,92 \\
\hline 0 & $\mathrm{AU}$ & 26,31 & $\mathrm{R}$ & 9,61 \\
\hline 12,12 & MR & 5,26 & MR & 9,61 \\
\hline 0 & $\mathrm{AU}$ & 15,79 & MR & 5,77 \\
\hline 0 & $\mathrm{AU}$ & 10,53 & MR & 3,85 \\
\hline 0 & $\mathrm{AU}$ & 10,53 & MR & 3,85 \\
\hline 0 & $\mathrm{AU}$ & 5,26 & MR & 1,92 \\
\hline 9,09 & MR & 5,26 & MR & 7,69 \\
\hline
\end{tabular}

$62 \%$ das espécies. Fatores favorecem esse índice tais como a geografia da trilha muito acidentada e íngreme; os níveis altitudinais (Tab. 2) superiores a $950 \mathrm{~m}$ que tornam a área adversa ao turismo, à agricultura, à caça e à construção de moradias; o ambiente constantemente úmido e sombrio e a disponibilidade de substrato adequado (Fig. 4). A trilha que vai da Vila do Abraão até a Vila Dois Rios apresenta o segundo maior índice de diversidade (Tab. 2). Esta é a única estrada da Ilha Grande e por isso é muito usada por moradores e turistas que trafegam entre os dois vilarejos. Frahm \& Gradstein (1991) comentam que barrancos de estradas abertos em baixas altitudes são rapidamente recobertos por numerosos táxons não encontrados em outras áreas de mesma altitude, explicando assim o índice de diversidade alto encontrado na trilha de Abraão - Vila Dois Rios que apresenta essas características físicas.

Segundo Gradstein \& Pócs (1989) cerca de 90\% das briófitas das florestas tropicais úmidas pertencem a 15 famílias: Callicostaceae, Calymperaceae, Dicranaceae, Fissidentaceae, Frullaniaceae, Hypnaceae, Lejeuneaceae, Lepidoziaceae, Meteoriaceae, Neckeraceae, Orthotrichaceae, Plagiochilaceae, Pterobryaceae, Radulaceae e Sematophyllaceae, todas representadas nas áreas estudadas (Tab. 1), mostrando que segue os padrões mencionados para as florestas tropicais úmidas.

Lejeuneaceae nas florestas tropicais de baixa altitude é a mais importante em termos de número de espécies seguida de Calymperaceae, Callicostaceae, Orthotrichaceae, Sematophyllaceae entre os musgos e de Lepidoziaceae, Plagiochilaceae e Frullaniaceae entre as hepáticas (Gradstein 1992). Nas áreas 


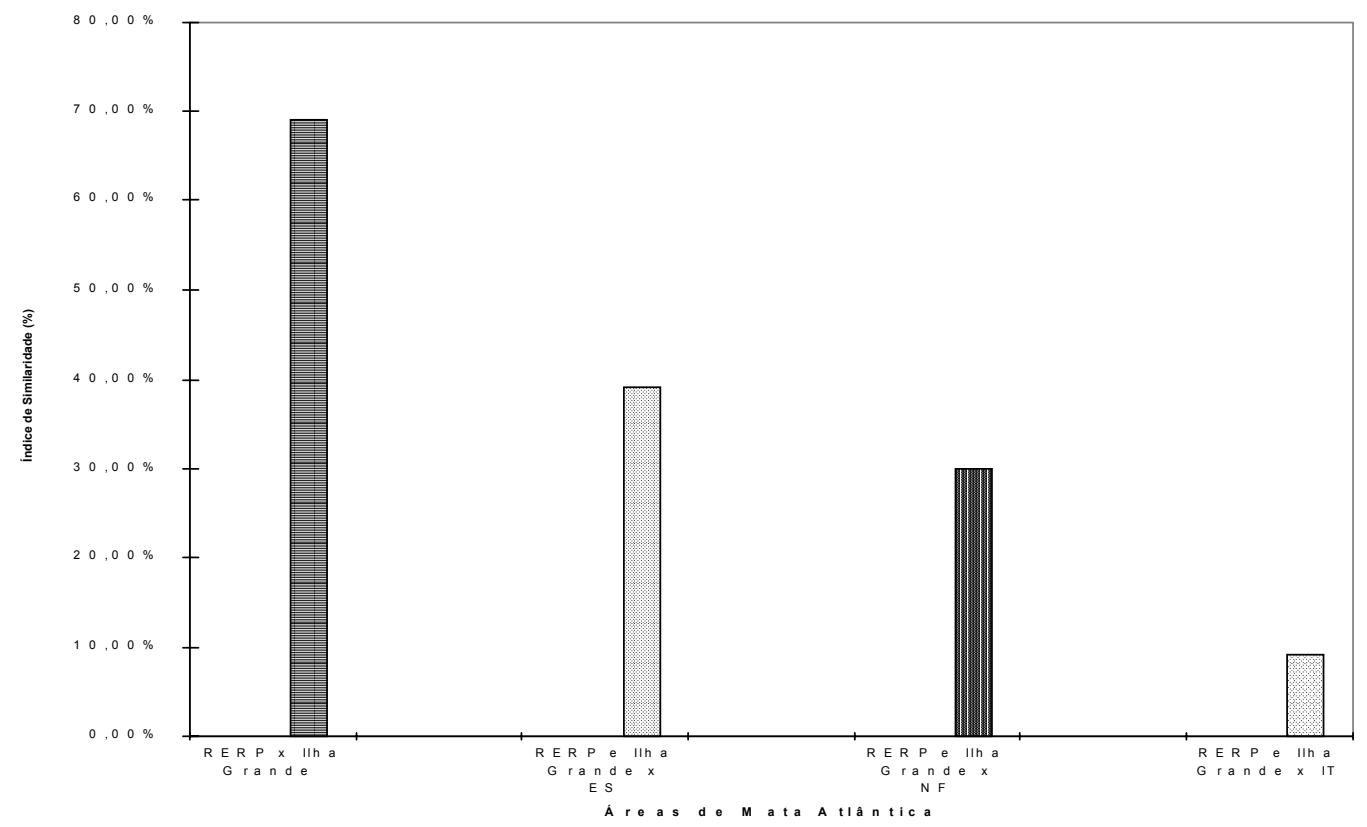

Fig. 2. Índice de similaridade entre a Reserva Ecológica de Rio das Pedras x Ilha Grande; Reserva Ecológica de Rio das Pedras e Ilha Grande x Espírito Santo (ES); Reserva Ecológica de Rio das Pedras e Ilha Grande x Nova Friburgo (NF); Reserva Ecológica de Rio das Pedras e Ilha Grande x Itatiaia (IT).

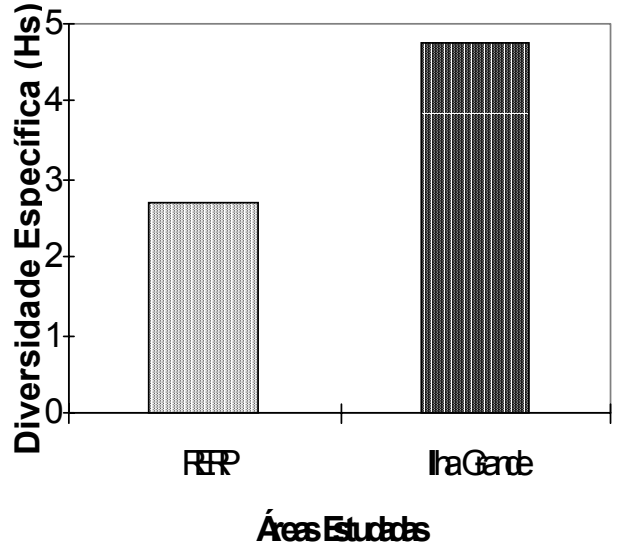

Fig. 3. Diversidade específica da Reserva Ecológica de Rio das Pedras e da Ilha Grande. estudadas foram encontradas 38 espécies de Lejeuneaceae, 13 de Frullaniaceae e Dicranaceae, 11 de Calymperaceae, dez de Orthotrichaceae e Fissidentaceae e nove de Meteoriaceae e Plagiochilaceae (Tab. 1). Almeida Sá (1995) também encontrou maior riqueza específica em Lejeuneaceae, Fissidentaceae, Calymperaceae e Sematophyllaceae, corroborando os dados obtidos nas áreas estudadas.

Segundo Gradstein \& Pócs (1989) cerca de 90\% das briófitas das florestas tropicais úmidas pertencem a 15 famílias: Callicostaceae, Calymperaceae, Dicranaceae, Fissidentaceae, Frullaniaceae, Hypnaceae, Lejeuneaceae, Lepidoziaceae, Meteoriaceae, Neckeraceae, Orthotrichaceae, Plagiochilaceae, Pterobryaceae, Radulaceae e Sematophyllaceae, todas representadas nas áreas estudadas (Tab. 1), mostrando que segue os padrões mencionados para as florestas tropicais úmidas.

Lejeuneaceae nas florestas tropicais de baixa altitude é a mais importante em termos de número 


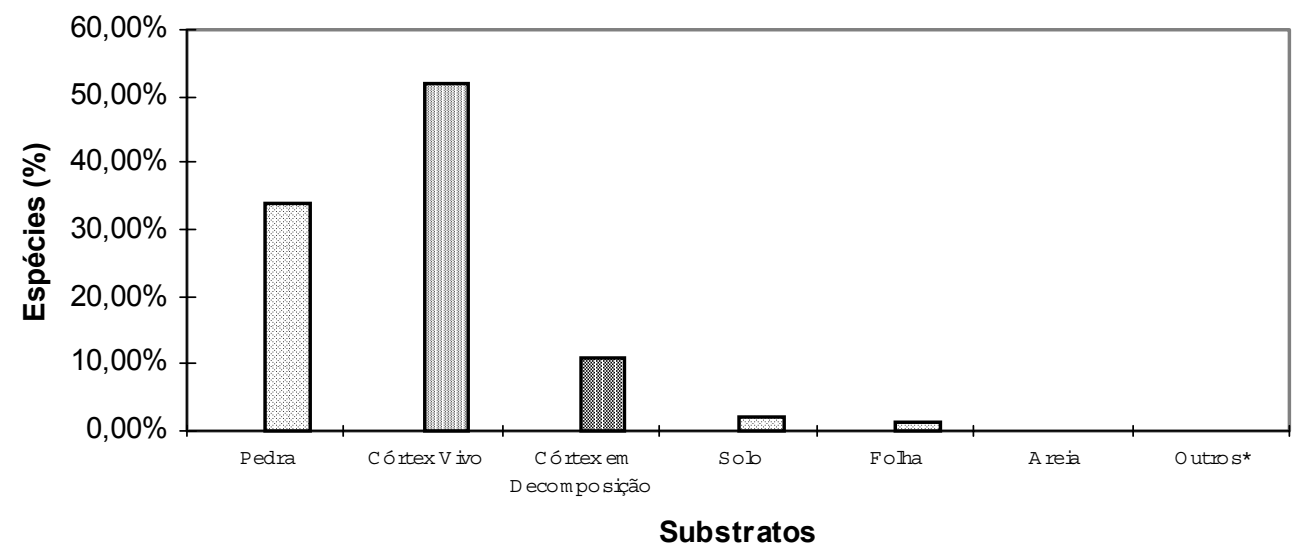

Fig. 4. Distribuição de espécies (antóceros, hepáticas e musgos) nos substratos encontrados na trilha do Pico do Papagaio. (Outros *: Canos de ferro, tijolos e basidiocarpos).

Tab. 2. Diversidade específica (Hs) e altitude (m) das trilhas da Ilha Grande, município de Angra dos Reis e da RERP, Município de Mangaratiba.

$\begin{array}{llllll}\text { Trilhas Ilha Grande } & \text { Hs } & \text { Altitude } & \text { Trilhas RERP } & \text { Hs } & \text { Altitude } \\ \text { Pico do Papagaio } & 4,3976 & 0-1100 & \text { Trilha } 14 & 3,3961 & 350-600 \\ \text { Abraão - Vila Dois Rios } & 4,3421 & 0-350 & \text { Trilha 13 } & 3,3687 & 350-500 \\ \text { Morro do Cristino } & 3,8878 & 150-500 & \text { Trilha 24 } & 3,3243 & 0-500 \\ \text { Parnaioca } & 3,8223 & 0-250 & \text { Trilha 32 } & 3,2417 & 150-450 \\ \text { Caxadasso } & 3,6706 & 0-200 & \text { Trilha 4 } & 2,9100 & 50-200 \\ \text { Saco do Céu } & 3,4907 & 0-200 & \text { Trilha 30 } & 2,9073 & 50-150 \\ \text { RBPS } & 3,351 & 0-200 & \text { Trilha 26 } & 2,8388 & 350-550 \\ \text { Praia do Mangue } & 3,2804 & 0-200 & \text { Trilha 7 } & 2,8167 & 50-100 \\ \text { Lazareto } & 3,1759 & 0-100 & \text { Trilha 18 } & 2,7897 & 50-100 \\ \text { Praia das Palmas } & 3,1597 & 0-200 & \text { Trilha 12 } & 2,7601 & 0-100 \\ \text { Japaris - Enseada da Estrela } & 3,0979 & 0-150 & \text { Trilha 8 } & 2,7338 & 0-200 \\ \text { Aqueduto } & 2,898 & 0-50 & \text { Trilha 20 } & 2,6226 & 0-150 \\ \text { Praia Preta } & 2,795 & 0-100 & \text { Trilha 10 } & 2,6126 & 0-100 \\ \text { Freguesia de Santana } & 2,6489 & 0-100 & \text { Trilha 11 } & 2,6105 & 0-100 \\ \text { Praia de Lopes Mendes } & 2,3749 & 0-50 & \text { Trilha 31 } & 2,5738 & 150-200 \\ \text { Abraãozinho } & 2,3272 & 0-50 & \text { Trilha Principal } & 2,5351 & 0-150 ? \\ \text { Aroeira } & 2,1441 & 0-50 & \text { Trilha 9 } & 2,4577 & 0-200 \\ \text { Trilhas RERP } & \text { Hs } & \text { Altitude } & \text { Trilha 3 } & 1,2388 & 0-150 \\ \text { Trilha 5 } & 3,938 & 150-200 & \text { Trilha 1 } & 1,0836 & 0-150 \\ \text { Trilha 16 } & 3,6068 & 100-350 & \text { Trilha 22 } & 0 & 0-150 \\ \text { Trilha 2 } & 3,4174 & 0-200 & & & \end{array}$


de espécies seguida de Calymperaceae, Callicostaceae, Orthotrichaceae, Sematophyllaceae entre os musgos e de Lepidoziaceae, Plagiochilaceae e Frullaniaceae entre as hepáticas (Gradstein 1992). Nas áreas estudadas foram encontradas 38 espécies de Lejeuneaceae, 13 de Frullaniaceae e Dicranaceae, 11 de Calymperaceae, dez de Orthotrichaceae e Fissidentaceae e nove de Meteoriaceae e Plagiochilaceae (Tab. 1). Almeida Sá (1995) também encontrou maior riqueza específica em Lejeuneaceae, Fissidentaceae, Calymperaceae e Sematophyllaceae, corroborando os dados obtidos nas áreas estudadas.

Segundo Schofield (1985) as briófitas das florestas tropicais úmidas não são ricas em diversidade e nem em abundância, entretanto Gradstein \& Pócs (1989) relatam que as florestas tropicais úmidas abrigam entre 25 e $30 \%$ do total de briófitas, o que corresponde a um número superior a qualquer outro ecossistema. Dajoz (1983) menciona que um ecossistema com um alto índice de diversidade específica se caracteriza por apresentar um grande número de espécies representadas por um pequeno número de indivíduos. Nas áreas estudadas a maioria das espécies apresenta uma freqüência relativa muito rara (Fig. 5 A, B e C), caracterizando uma diversidade alta. As espécies muito abundantes, comoLejeunea glaucescens, Plagiochila rutilans e Sematophyllum subpinnatum (Tab. 1), pertencem à famílias com maior freqüência em matas tropicais úmidas (Gradstein \& Pócs 1989).

\section{3- Freqüência absoluta}

Foi calculada a freqüência absoluta das 231 espécies coletadas nos dois municípios (Fig. 5 A); do total das espécies coletadas na RERP, município de Mangaratiba (Fig. 5 B) e do total das espécies coletadas na Ilha Grande, município de Angra dos Reis (Fig. 5 C).

Seis classes de freqüência foram determinadas: muito abundante $(78,85-66,03 \%$ nos dois municípios; 84,84-71,20\% na RERP; 79,95$67,50 \%$ na Ilha Grande), abundante $(66,02-$ $53,20 \%$ nos dois municípios; 71, 19-57,55\% na RERP; $67,49-55,04 \%$ na Ilha Grande), freqüente (53,19-40,37\% nos dois municípios; 57,5443,91\% na RERP; 55,03-42,58\% na Ilha Grande), pouco freqüente $(40,36-27,54 \%$ nos dois municípios; $43,26-30,3 \%$ na RERP; 42,57$30,12 \%$ na Ilha Grande), rara $(27,53-14,71 \%$ nos dois municípios; 30,25-16,61\% na RERP; 30,11$17,66 \%$ na Ilha Grande) e muito rara $(14,70$ $1,88 \%$ nos dois municípios; $16,60-2,93 \%$ na RERP; 17,65-5,20\% na Ilha Grande).

A maioria das espécies apresenta uma freqüência absoluta muito rara enquanto poucas como Plagiochila rutilans, Lejeunea glaucescens, Sematophyllum subpinnatum são muito abundantes (Tab. 1 e Fig. 5 A, B, C).

\section{4 - Substrato}

Segundo Schofield (1985) as briófitas diferem das plantas com sementes no que diz respeito à tolerância ecológica, porque são capazes de colonizar superfícies duras como o córtex das árvores e as rochas. Em florestas tropicais úmidas, o substrato mais favorável ao estabelecimento de briófitas é o córtex vivo seguido do córtex em decomposição (Pócs 1982 e Germano \& Pôrto 1996).

Nas áreas estudadas o substrato mais freqüente entre os antóceros e hepáticas são as pedras seguidas do córtex vivo, córtex em decomposição, solo, outros (cano de ferro, tijolos e basidiocarpos) (Fig. 6-A e Tab. 3). Entre os musgos são as pedras, seguidas do córtex vivo, córtex em decomposição, solo, outros (cano de ferro, tijolos e basidiocarpos), folhas e areia (Fig. 6-B). A mesma observação foi feita por Almeida Sá (1995) que obteve maior representatividade de briófitas sobre rochas, seguidas de córtex em decomposição, córtex vivo e solo.

Dentre os musgos, 42 crescem em um só tipo de substrato, 39 em dois e 49 em mais de dois substratos, enquanto que dos antóceros e hepáticas 41 crescem em um só tipo de substrato, 30 em dois e 29 em mais de dois caracterizandoos como mais seletivos (Tab. 3). Bazzania pallide-virens coletada em um só substrato na Ilha Grande (Tab. 3), aparece crescendo sobre córtex vivo e em decomposição na Amazônia (Lisboa 1976). No entanto Calypogeia miquelli cresce em apenas um tipo de substrato, tanto nas áreas estudadas como na Reserva Mocambo, no Pará (Lisboa 1985).

Lejeunea flava, L. glaucescens, Bryum paradoxum, Helicodontium capillare e Vesicularia vesicularis 
(A)

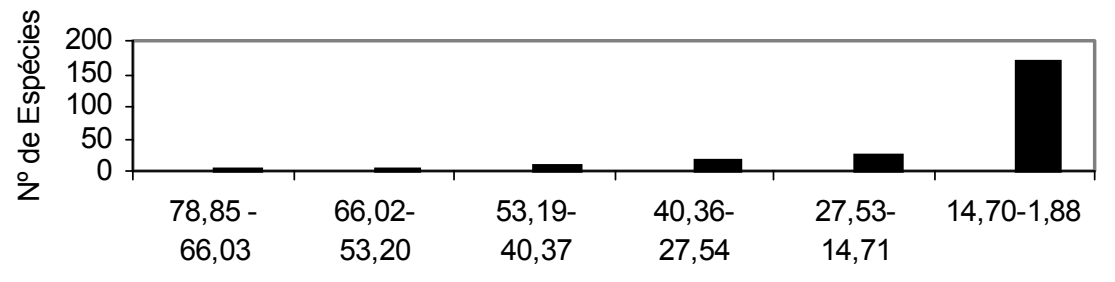

Classes de Freqüência

(B)

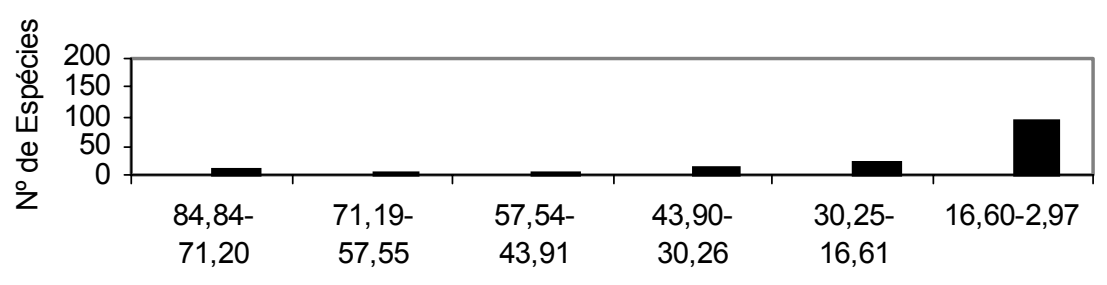

Classes de Freqüência (\%)

(C)

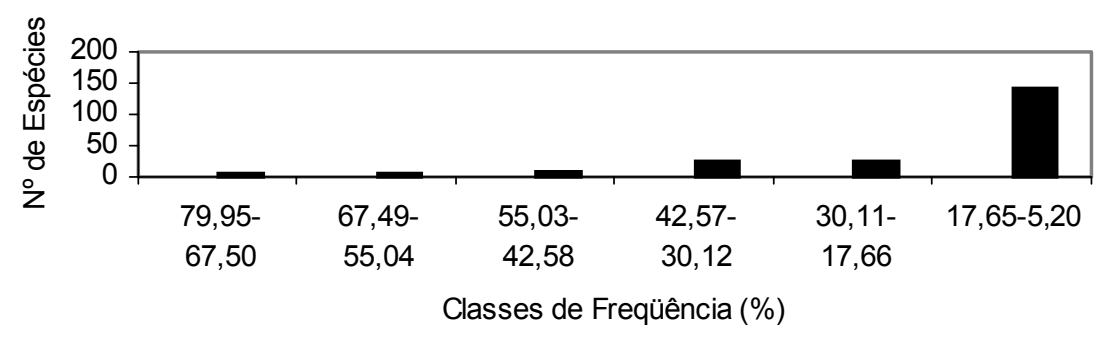

Figura 5. Freqüência absoluta das espécies coletadas (A) nos dois municípios, (B) na RERP, no município de Mangaratiba e (C) na Ilha Grande, município de Angra dos Reis.

são as que apresentam maior variedade de substratos (Tab. 3). Dessas espécies Bryum paradoxum apresenta uma freqüência absoluta inferior a $30 \%$, isto é, foram coletados poucos exemplares porém em substratos variados. Almeida Sá (1995) também cita Vesicularia vesicularis como uma espécie euritópica.

Bryum densifolium, Campylopus trachyblepharon e Fissidens diplodus são as únicas espécies que crescem sobre areia de praia (Tab. 3). Das espécies coletadas por Yano \& Costa (1993) na Restinga de Massambaba (RJ), somente Campylopus arenaceum cresce no solo arenoso, o mesmo tendo sido observado por Behar et al. (1992) para a Restinga de Setiba (ES). As espécies em questão, apresentam características que, segundo Proctor (1979), auxiliam a distribuição e o armazenamento de água da chuva, permitindo o crescimento em ambiente árido, como o formato alongado das células superiores, o ápice excurrente e a presença de pontuações nas células basais dos filídios de $B$. densifolium; o ápice acuminado, as lamelas dorsais e as células basais hialinas de C. trachyblepharon; e as células papilosas dos filídios de $F$. diplodus. 
A

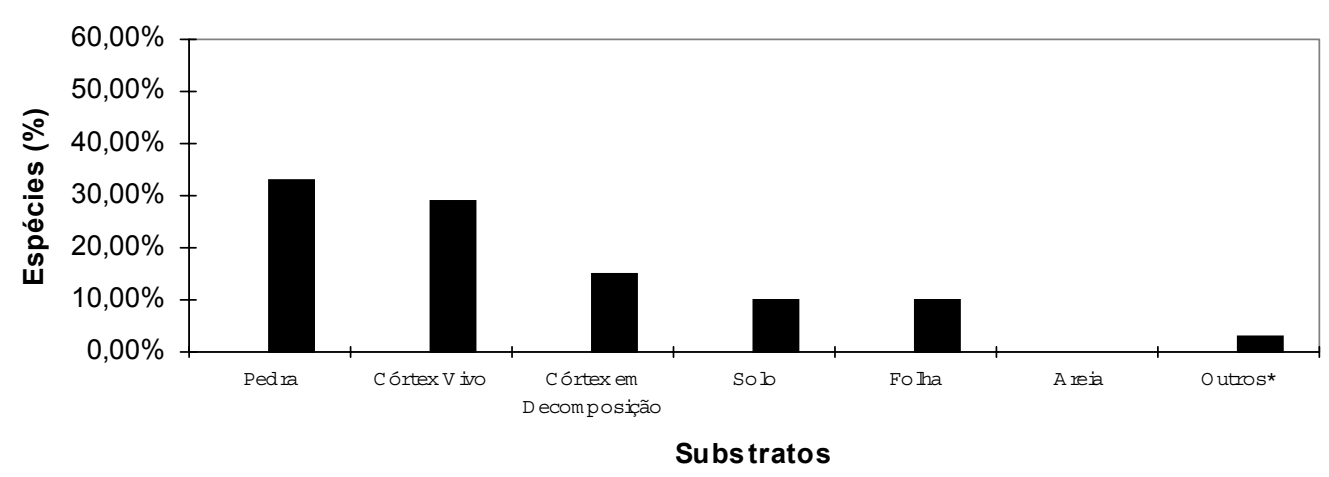

B

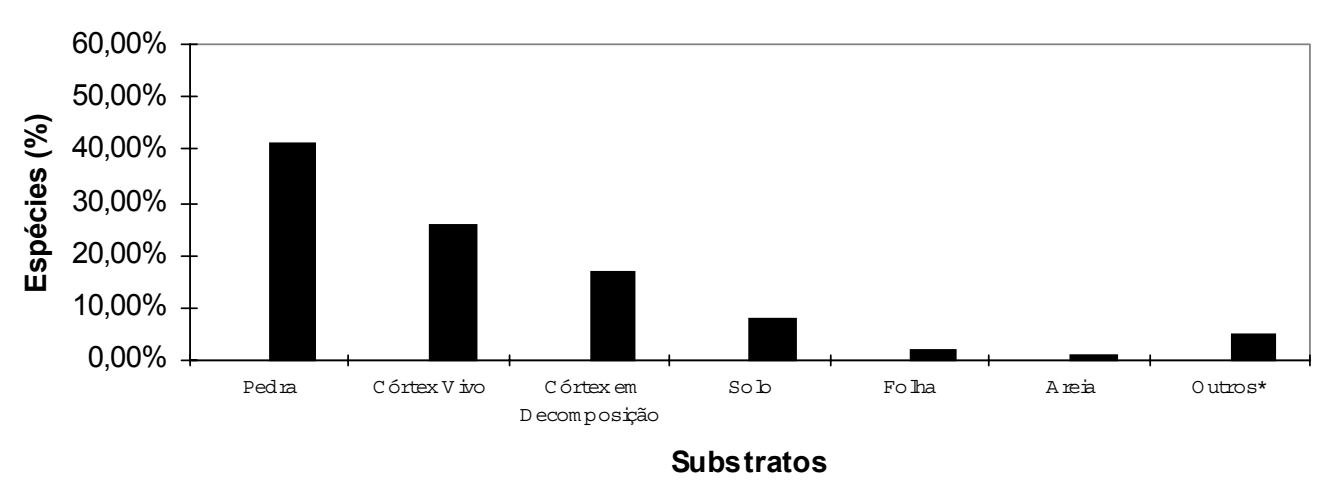

Figura 6. Distribuição das espécies nos diferentes tipos de substratos encontrados nas áreas estudadas. A) Antóceros e hepáticas. B) Musgos. (Outros *: Canos de ferro, tijolos, basidiocarpos).

Octoblepharum albidum embora cresça sobre grande variedade de substratos (Tab. 3) na maioria das coletas foi encontrado crescendo sobre o córtex de palmeiras. Yano (1992b) observou que a espécie cresce sobre córtex vivo ou em decomposição, sobre pedra e xaxim e que na região amazônica cresce abundante e preferencialmente sobre troncos de palmeiras. Dumortiera hirsuta e Monoclea gottschei ssp. elongata crescem exclusivamente sobre rochas (Tab. 3), o que também foi observado por Almeida Sá (1995). Segundo Giminghan \& Birse (1957) o substrato tem grande influência no tipo do arranjo das leivas, assim, sobre rochas crescem preferencialmente espécies que formam tapetes lisos como o das hepáticas talosas.

Anthoceros punctatus, Calypogeia miquelii, Isotachis aubertii, Kurzia capillaris var. verrucosa, Omphalanthus filiformis, Barbula indica, Polytrichum brasiliensis e Trematodon longicollis, espécies muito raras (Tab. 1), foram coletadas crescendo somente sobre barrancos ou solo (Tab. 3), substratos que após a colonização inicial pelas briófitas é invadido rapidamente pelas plantas vasculares (Schofield 1985). Richards (1984) atribui a reduzida ocorrência de briófitas sobre o solo das matas tropicais úmidas à grande quantidade de folhas que caem e recobrem 
A

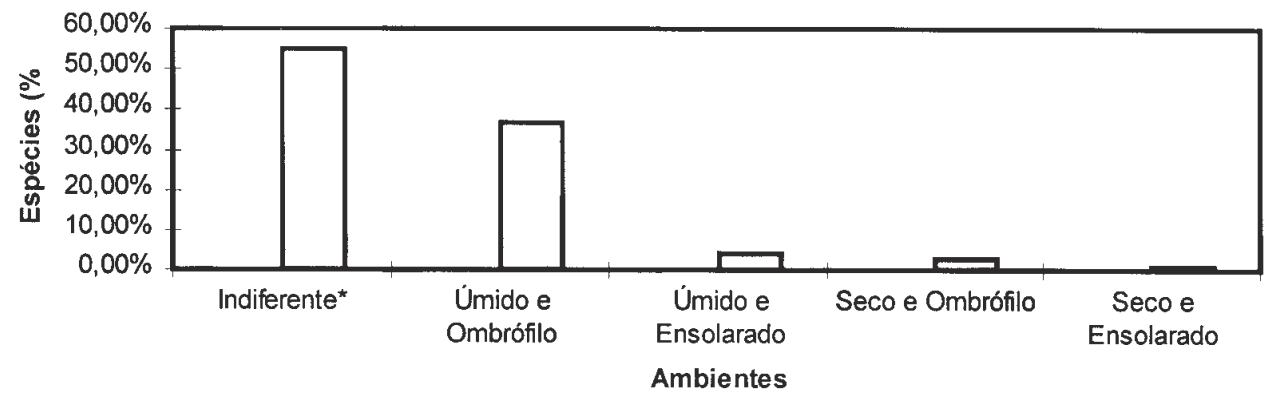

B

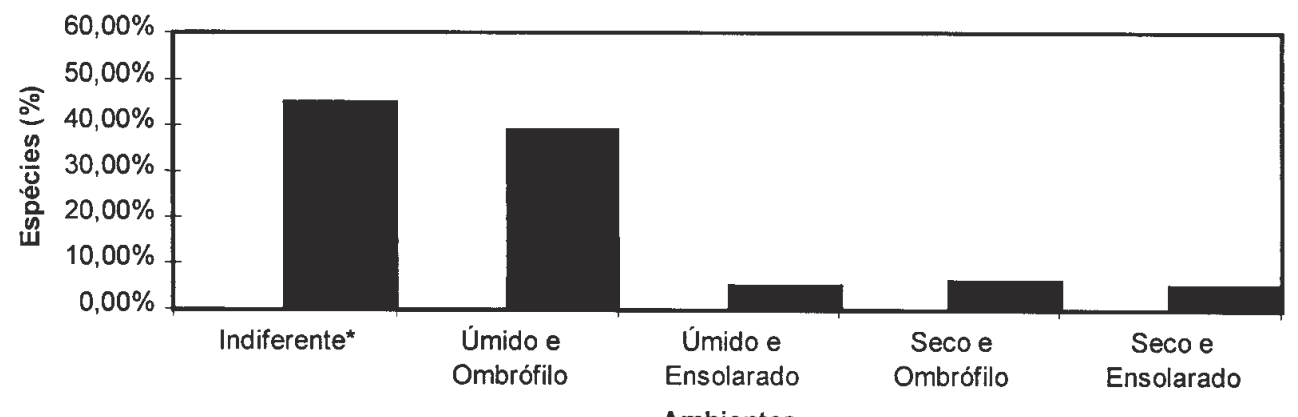

Figura 7. Distribuição das espécies pelos diferentes ambientes encontrados nas áreas estudadas. A) Antóceros e hepáticas. B) Musgos. (Indiferentes* Espécies que foram encontradas em todos os tipos de ambientes encontrados).

esse substrato. Gradstein \& Pócs (1989) relatam que espécies terrestres são encontradas principalmente em solo exposto e perturbado, como os cortes de estradas.

Comunidades de briófitas epífilas constituem uma característica das florestas tropicais úmidas, raramente ocorrendo fora desse habitat (Richards 1984). O número de espécies epífilas é pequeno embora seja comum coletar mais de 50 espécies em uma pequena área de mata úmida nos neotrópicos (Richards 1984). Segundo Schofield (1985) nas florestas tropicais úmidas, as briófitas que crescem sobre folhas estão principalmente entre as Jungermanniales. Nas áreas estudadas
$81 \%$ das espécies epífilas são hepáticas de Lejeuneaceae, sendo que dessas, Cololejeunea obliqua, Colura tortifolia, Diplasiolejeunea brunea, D. pellucida, Drepanolejeunea mosenii, Leptolejeunea elliptica e L. moniliata, foram encontradas crescendo somente nesse substrato (Tab. 3).

\section{5 - Ambiente}

A maioria das briófitas prefere ambiente úmido e sombrio, entretanto somente $37 \%$ dos antóceros e hepáticas (Fig. 7 - A) e 39\% dos musgos (Fig. 7 - B) apresentam esse habitat como único, sendo que 55\% dos antóceros e hepáticas (Fig. 7 - A) e 
Tab. 3. Distribuição das espécies nos diferentes substratos $($ Corticícola $=\mathrm{C}$; Epixílico $=\mathbf{E}$; Foliícola $=\mathbf{F}$; Rupícola $=\mathbf{R}$; Terrícola $=\mathbf{T}$ e $\mathbf{T}^{*}=$ Arreia da praia; Outros $=\mathbf{O}$ (cano de ferro, tijolos e basidiocarpos), ambientes (Ensolarado $=\mathrm{EN}$; Ombrófilo $=$ OM; Seco $=\mathrm{SC}$; Submerso $=$ SB e Umbrófilo $=$ UM) e altitudes nas áreas de estudo (continua).

Famílias e Espécies coletadas

Altitude

ANTHOCEROTACEAE

Anthoceros punctatus L.

Phaeoceros laevis (L.) Prosk.

HERBERTACEAE

Herbertus angustivittatus (Steph.) Fulf.

$H$. divergens (Steph.) Herz.

TRICHOCOLEACEAE

Trichocolea flaccida (Spruce) Spruce

LEPIDOZIACEAE

Arachniopsis diacantha (Mont.) Howe

Bazzania pallide-virens (Steph.) Fulf.

B. stolonifera (Sw.) Trev.

Kurzia brasiliensis (Steph.) Grolle

K. capillaris (Sw.) Grolle var. verrucosa

(Steph.) Pócs

Lepidozia inaequalis (Lehm. \& Lindenb.)

Gott. et al.

Telaranea nematodes (Gott. ex Aust.) Howe

CALYPOGEIACEAE

Calypogeia miquelii Mont.

CEPHALOZIELLACEAE

Kymatocalyx dominicensis (Spruce) Váña GEOCALYCACEAE

Lophocolea bidentata

L. martiana Nees

PLAGIOCHILACEAE

Plagiochila distinctifolia Lindenb.

P. kroneana Steph.

P. martiana (Nees) Lindenb.

P. montagnei Nees

P. rutilans Lindenb.

P. scissifolia Steph.

P. subplana Lindenb.

Plagiochila sp 1.

Plagiochila sp 2

BALANTIOPSIDACEAE

Isotachis aubertii (Schwaegr.) Mitt.

RADULACEAE.

Radula javanica Gott.

R. marginata (Tayl.) Mitt.

R. mexicana Lindenb. \& Gott.

PORELLACEAE

Porella brasiliensis (Raddi) Schiffn.

FRULLANIACEAE

Frullania arecae (Spreng.) Gott.

F. beyrichiana (Lehm. \& Lindenb.)

Lehm. \& Lindenb.

\begin{tabular}{|c|c|c|c|}
\hline Substratos & Umidade & \multicolumn{2}{|c|}{ Luminosidade } \\
\hline $\mathrm{T}$ & $\mathrm{OM}$ & - & 0 \\
\hline R-T & $\mathrm{OM}-\mathrm{SB}$ & EN-UM & $110-300$ \\
\hline $\mathrm{C}$ & - & - & 140 \\
\hline $\mathrm{R}$ & OM & OM & $840-1110$ \\
\hline $\mathrm{C}$ & - & - & 1110 \\
\hline $\mathrm{R}$ & - & UM & 240 \\
\hline $\mathrm{C}$ & - & UM & 110 \\
\hline C-R & OM & UM & 890 \\
\hline R-C-E & $\mathrm{OM}$ & UM-EN & $450-900$ \\
\hline $\mathrm{T}$ & OM & UM & 300 \\
\hline E-R & $\mathrm{OM}$ & EN & $600-900$ \\
\hline T-R & $\mathrm{OM}$ & - & 240 e 1010 \\
\hline $\mathrm{T}$ & OM & UM-EN & $220-340$ \\
\hline T-R & $\mathrm{OM}$ & UM-EN & $160-320$ \\
\hline $\mathrm{R}$ & - & - & 50 \\
\hline R-C-E-T & OM & UM & $0-380$ \\
\hline C-R & $\mathrm{OM}$ & EN & 840 \\
\hline $\mathrm{R}-\mathrm{C}$ & SB-OM & UM & 350 \\
\hline $\mathrm{R}-\mathrm{C}$ & $\mathrm{OM}$ & UM-EN & 300 \\
\hline C-E-R-T & SB-OM & UM & $0-1110$ \\
\hline R-C-E-O & OM-SB & UM-EN & $0-1110$ \\
\hline R-C-E & OM-SC & UM-EN & $180-420$ \\
\hline $\mathrm{R}-\mathrm{C}$ & $\mathrm{OM}$ & UM & $240-320$ \\
\hline R-E & $\mathrm{OM}$ & - & 210 \\
\hline $\mathrm{R}$ & $\mathrm{OM}$ & - & 210 \\
\hline $\mathrm{T}$ & OM-SC & UM & $120-300$ \\
\hline $\mathrm{R}-\mathrm{C}$ & $\mathrm{OM}$ & UM & $130-650$ \\
\hline $\mathrm{E}$ & - & - & $600-1110$ \\
\hline $\mathrm{C}$ & - & - & $600-1110$ \\
\hline $\mathrm{R}-\mathrm{C}$ & OM-SC & UM-EN & 320 \\
\hline $\mathrm{R}$ & - & - & $450-500$ \\
\hline R-T-E-C & $\mathrm{OM}$ & UM-EN & $30-1110$ \\
\hline
\end{tabular}

TROPICAL BRYOLOGY 22 (2002) 
F. brasiliensis Raddi

$F$. caulisequa (Nees) Nees

F. dilatata (L.) Dum.

$F$. ericoides (Nees) Nees

$F$. montagnei Gott.

$F$. neesii Lindenb.

F. riojaneirensis (Raddi) Aongstr.

$F$. setigera Steph.

F. supradecomposita (Lehm. \& Lindenb.)

Lehm. \& Lindenb

\section{LEJEUNEACEAE}

Acrolejeunea emergens (Mitt.) Steph. Aphanolejeunea diaphana (Evans) Schust. Archilejeunea parviflora (Nees) Schiffn.

Bryopteris diffusa (Sw.) Nees

B. filicina (Sw.) Nees

Caudalejeunea lehmanniana (Gott.) Steph.

Ceratolejeunea cubensis (Mont.) Schiffn.

C. rubiginosa Steph.

Cheilolejeunea rigidula (Nees ex Mont.) Schust.

C. trifaria (Reinw. et al.) Mizut.

Cololejeunea obliqua (Nees \& Mont.) S. Arell

Colura tortifolia (Mont. ex Nees) Trev.

Diplasiolejeunea brunnea Steph.

D. pellucida (Meiss.) Schiffn.

Drepanolejeunea mosenii (Steph.) Bischler

D. orthophylla (Nees \& Mont.) Bischler

Harpalejeunea uncinata Steph.

Lejeunea bermudiana (Evans) Schust.

L. caespitosa Lindb. ex G.L. \& Nees

L. cancellata Nees \& Mont.

L. flava (Sw.) Nees

L. glaucescens Gott.

L. laete-virens Nees \& Mont. ex Mont.

L. minutiloba Evans

L. pterogonia (Lehm. \& Lindenb.) Mont.

Leptolejeunea elliptica (Lehm. \& Lindenb.)

Schiffn.

L.moniliata Steph.

Leucolejeunea unciloba (Lindenb.) Evans

L. xanthocarpa (Lehm. \& Lindenb.) Evans

Lopholejeunea nigricans (Lindenb.) Schiffn.

L. subfusca (Nees) Steph.

Marchesinia brachiata (Sw.) Schiffn.

Omphalanthus filiformis (Sw.) Nees

Rectolejeunea phyllobola (Nees \& Mont.) Evans

R. pililoba (Spruce) Schust

Schiffneriolejeunea polycarpa (Nees) Gradst.

Stictolejeunea squamata (Willd. ex Web.) Schiffn. R

Symbiezidium transversale (Sw.) Trev.

FOSSOMBRONIACEAE

Fossombronia foveolata S. O. Lindb.

PALLAVICINIACEAE

Symphyogyna aspera Steph. ex McCormick

S. brasiliensis Nees \& Mont.

$\begin{array}{llll}\text { R-C-E } & \text { OM-SC } & \text { UM-EN } & 0-1110 \\ \text { R-C-E } & \text { OM-SC } & \text { UM } & 40-1110 \\ \text { R } & \text { OM-SC } & - & 240 \\ \text { C-E-R } & \text { OM } & \text { UM } & 0-480 \\ \text { C } & \text { OM-SC } & - & 840-1110 \\ \text { C-E-R } & \text { OM-SC } & - & 0-1110 \\ \text { C-E-R } & \text { SC } & \text { UM-EN } & 40-400 \\ \text { C } & - & - & 840-1110\end{array}$

C-R SC UM-EN $\quad 0-940$

$\begin{array}{llll}\mathrm{C} & \mathrm{OM}-\mathrm{SC} & \mathrm{UM} & 0-60\end{array}$

$\begin{array}{lll}\text { F-C } & \text { OM } & \text { UM }\end{array}$

$\begin{array}{llll}\mathrm{R} & \mathrm{OM} & \mathrm{EN} & 40\end{array}$

C-E OM-SC $\quad$ EN $50-1110$

C-E-R OM-SC UM-EN 40-1110

$\begin{array}{llll}\mathrm{C}-\mathrm{C} & \mathrm{OM} & \mathrm{UM}-\mathrm{EN} & 20\end{array}$

C-R-O OM-SC UM-EN 50-100

C-R-T-O OM-SC UM-EN 40-38

C-E-R-O OM-SC UM-EN $80-240$

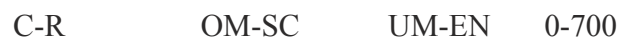

F OM-SC UM $40-80$

$\begin{array}{llll}\mathrm{F} & \mathrm{OM} & - & 50\end{array}$

$\begin{array}{llll}\mathrm{F} & - & - & 50-1110\end{array}$

$\begin{array}{llll}F & \text { OM } & \text { UM } & 600\end{array}$

$\begin{array}{llll}\mathrm{F} & - & - & 600\end{array}$

$\begin{array}{llll}\text { F-C-E } & \text { OM } & - & 150-320\end{array}$

$\begin{array}{llll}\text { F-C } & \text { OM } & \text { EN } & 100-150\end{array}$

$\begin{array}{llll}\mathrm{C} & - & - & -\end{array}$

$\begin{array}{llll}\text { F-C-R } & \text { OM } & \text { UM-EM } & 100-900\end{array}$

$\begin{array}{llll}C & \text { SC } & \text { EN } & 150\end{array}$

F-C-E-R-T OM-SC UM-EN 0-1110

F-C-E-R-O OM-SC UM-EN 0-1110

C-E-R OM-SC UM-EN 0-480

$\begin{array}{llll}\text { F-C-E-R } & \text { SC } & \text { UM } & 0-880\end{array}$

$\begin{array}{lll}\text { R-F-C OM } & \text { UM-EN } 350\end{array}$

$\begin{array}{llll}\mathrm{F} & - & - & 50\end{array}$

F OM-SC UM-EN 250-300

$\begin{array}{llll}\mathrm{C} & - & - & -\end{array}$

C-R $\quad$ SC $\quad$ UM $200-1110$

$\begin{array}{llll}\text { C-E-R } & \text { OM } & \text { UM } & 150-240\end{array}$

C-R OM-SC UM $0-140$

C-R OM-SC UM-EN $\quad 0-150$

$\begin{array}{llll}\text { T } & \text { OM } & \text { UM } & 280-320\end{array}$

$\begin{array}{llll}\mathrm{R} & \mathrm{OM} & - & 240\end{array}$

$\begin{array}{llll}\text { F-C-R } & \text { OM-SC } & - & 10\end{array}$

C-E OM-SC UM-EN $\quad 0-400$

OM-SC UM 100-160

$\begin{array}{llll}\mathrm{C} & - & - & 50\end{array}$

$\begin{array}{llll}\mathrm{R} & \mathrm{OM} & - & 50\end{array}$

R-C-T OM-SC UM-EN 20-380

R-T OM-SC-SB UM-EN $0-420$ 


\section{ANEURACEAE}

Riccardia alata (Steph.) Hell

$R$. chamedryfolia (With.) Grolle

$R$. metzgeriiformis (Steph.) Hell

$R$. regnellii (Aongstr.) Hell

METZGERIACEAE

Metzgeria albinea Spruce

M. angusta Steph.

M. aurantiaca Steph.

M. convoluta Steph.

M. dichotoma (Sw.) Nees

M. furcata (L.) Corda

MONOCLEACEAE

Monoclea gottschei Lindb. ssp. elongata Gradst. \& Mues

20-420

WIESNERELLACEAE

Dumortiera hirsuta (Sw.) Nees

MARCHANTIACEAE

Marchantia berteroana Lehm. \& Lindenb.

M. chenopoda $\mathrm{L}$.

M. papillata Raddi

POLYTRICHACEAE

Pogonatum pensilvanicum (Hedw.) P. Beauv.

Polytrichum brasiliense Hampe

P. commune Hedw.

$P$. juniperinum Hedw.

ORTHOTRICHACEAE

Groutiella tomentosa (Hornsch.) Wijk \& Marg.

G. tumidula (Mitt.) Vitt

Macrocoma frigidum (C. Muell.) Vitt

Macromitrium argutum Hampe

M. microstomum (Hook. \& Grev.) Schwaegr.

M. pellucidum Mitt.

M. punctatum (Hook. \& Grev.) Brid.

$M$. richardii Schwaegr.

Schlotheimia jamesonii (Arnott) Brid.

S. rugifolia (Hook.) Schwaegr.

ERPODIACEAE

Erpodium glaziouii Hampe

HELICOPHYLLACEAE

Helicophyllum torquatum (Hook.) Brid.

BRYACEAE

Brachymenium systylium (C. Muell.) Jaeg.

Bryum argenteum Hedw.

$B$. billardieri Schwaegr.

$B$. densifolium Brid.

B. limbatum C. Muell.

B. matto-grossense Broth.

B. pabstianum C. Muell.

B. paradoxum Schwaegr.

B. renauldii Röll ex Ren. \& Card.

PLAGIOMNIACEAE

Plagiomnium rhynchophorum (Hook. f.) T. Kop. R-C

MNIACEAE

Pyrrhobryum spiniforme (Hedw.) Mitt.

$\begin{array}{ll}\text { R } & \text { OM } \\ \text { T-R } & \text { OM } \\ \text { T-R-E } & \text { OM } \\ \text { T-R-E-C } & \text { OM } \\ & \\ \text { T-R-C } & \text { OM } \\ \text { C-R-F } & \text { OM } \\ \text { C-R } & \text { OM } \\ \text { C-E-R } & \text { OM-SC } \\ \text { C } & \text { OM-SC } \\ \text { C-R } & \text { OM-SC }\end{array}$

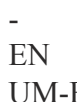

UM-EN $\quad 0-280$

UM-EN $\quad 140-320$

UM 260-400

UM $\quad 310-360$

UM $\quad 100-650$

UM 320-460

UM-EN 190-450

$\mathrm{R}$

OM-SB UM

\section{$\mathrm{R}$}

$\mathrm{R}$

$\mathrm{R}$

R-T

R-T

$\mathrm{T}$

R-T

R-T

$\mathrm{R}$

$\mathrm{R}$

C

C-E

C

R-C-E

$\mathrm{R}-\mathrm{C}$

R-C-E

$\mathrm{R}$

R-C-E

E

$\mathrm{R}$

T-R-C

R-C

R-C

$\mathrm{R}-\mathrm{T}^{*}$

$\mathrm{R}$

E

$\mathrm{R}$

R-T-C-E-F

$\mathrm{R}$

R-C-E
OM-SB

OM

OM

OM

OM-SC

$\mathrm{OM}$

OM-SC

OM-SC

OM-SC

OM-SC

$\mathrm{OM}$

OM

OM

OM-SC

OM-SC

OM-SC

OM-SC

OM-SC

UM

$0-420$

UM

UM

1000

$\begin{array}{ll}- & 160\end{array}$

UM-EN 280-320

EN $\quad 40$ e 1110

UM-EN 20-240

UM-EN 20-60

UM $\quad 0-910$

UM 620-1110

UM $\quad 990$

UM-EN 0-1110

UM-EN 20-1110

UM-EN 0-160

UM-EN $\quad 0-140$

UM 20-880

-
$-\quad 320$

UM-EN 20-240

UM-EN 30-180

EN 330-1010

EN 20

UM $\quad 0-280$

- $\quad 170$

EN 0-250

EN 20

UM-EN $\quad 0-500$

EN 0

$\mathrm{OM}$

$\mathrm{OM}$

UM

20-200

OM-SC

UM

$300-870$
UM-EN 0-220

- $\quad 320$ 
RHIZOGONIACEAE

Hymenodon aeruginosus (Hook. f. \& Wils.)

C. Muell.

BARTRAMIACEAE

Philonotis hastata (Duby) Wijk \& Marg.

P. uncinata (Schwaegr.) Brid.

THUIDIACEAE

Haplocladium microphyllum (Hedw.) Broth.

Thuidium tomentosum Besch.

RACOPILACEAE

Racopilum tomentosum (Hedw.) Brid.

MYRINIACEAE

Helicodontium capillare (Hedw.) Jaeg.

BRACHYTHECIACEAE

Aerolindigia capillacea (Hornsch.) Menzel

Steerecleus scariosus (Tayl.) Robins.

STEREOPHYLLACEAE

Eulacophyllum cultelliforme (Sull.)

Buck \& Ireland

Pilosium chlorophyllum (Hornsch.) C. Muell.

Stereophyllum radiculosum (Hook.) Mitt.

HYPNACEAE

Isopterygium tenerifolium Mitt.

Mittenothamnium elegantulum (Hook.) Card.

Vesicularia vesicularis (Schwaegr.) Broth.

ENTODONTACEAE

Entodon beyrichii (Schwaegr.) C. Muell.

Erythrodontium longisetum (Hook.) Par.

SEMATOPHYLLACEAE

Acroporium longirostre (Brid.) Buck

Sematophyllum adnatum (Michx.) Britt.

S. demissum (Wils.) Mitt.

S. subpinnatum (Brid.) Britt.

S. subsimplex (Hedw.) Mitt.

Taxithelium planum (Brid.) Mitt.

Trichosteleum fluviale (Mitt.) Jaeg.

LESKEACEAE

Herpetineuron toccoae (Sull. \& Lesq.) Card.

FABRONIACEAE

Fabronia ciliaris (Brid.) Brid. var. wrightii

(Sull. ex Sull.) Buck

LEUCODONTACEAE

Pseudocryphaea domingensis (Spreng.) Buck PTEROBRYACEAE

Calyptothecium duplicatum (Schwaegr.) Broth.

Jaegerina scariosa (Lor.) Arz.

Pireella cymbifolia (Sull.) Card.

Pterobryon densum (Schwaegr.) Hornsch.

METEORIACEAE

Floribundaria usneoides (Broth.) Broth.

Papillaria deppei (C. Muell.) Jaeg.

$P$. nigrescens (Hedw.) Jaeg.

Pilotrichella flexilis (Hedw.) Aongstr.

Orthostichella pentasticha (Brid.) Buck

Squamidium leucotrichum (Tayl.) Broth.

$\mathrm{R}$

T-C

T-C-R

R-C-E

OM-SC

$\mathrm{OM}$

UM

210

R-C-E-T

OM-SC

UM

$60-470$

C-E-R-F-O

OM-SC-SB

UM-EN

$0-400$

R-C-F-O

OM-SC

UM

20-340

R-T-C-E

OM-SC

UM-EN

20-400

R-C-E-O

OM-SC

UM

0-90

$\mathrm{R}$

$\mathrm{OM}$

UM

$120-580$

R-C-E OM-SC

UM-EN 20-540

R-T-C-E-O OM-SC

UM-EN

UM-EN

$0-480$

$0-500$

$\mathrm{R}$

$\mathrm{R}$

SC

C-E-T

$\mathrm{R}$

$\mathrm{R}$

R-C-E-O

R-C-E-T

R-E-C

E

$\mathrm{R}$

$\mathrm{R}$

R-C

$\mathrm{OM}$

UM-EN

0

$\mathrm{R}$

$\mathrm{SC}$

EN

0

C

C

$\mathrm{C}$

C-O

$-$

$\mathrm{SC}$

SC

UM

EN

260

E-R

R-C

C-R

C-R

OM-SC

OM-SC

$\mathrm{UM}$

OM-SC UM-EN 40-320

$\mathrm{OM}$

R-C-E OM-SC

220-1100

C-R

OM-SC

UM-EN $\quad 10-8900$

UM-EN $\quad 0-940$

TROPICAL BRYOLOGY 22 (2002) 
S. nigricans (Hook.) Broth.

Zelometeorium patulum (Hedw.) Manuel

Z. recurvifolium (Hornsch.) Manuel

NECKERACEAE

Neckeropsis disticha (Hedw.) Kindb.

N. undulata (Hedw.) Reichardt

N. villae-ricae (Besch.) Broth.

Porothamnium flagelliferum (C. Muell.) Fleisch.

Porotrichum substriatum (Hampe) Mitt..

PHYLLOGONIACEAE

Phyllogonium viride Brid.

PILOTRICHACEAE

Pilotrichum evanescens (C. Muell.) C. Muell.

CALLICOSTACEAE

Callicostella merkelii (Hornsch.) Jaeg.

C. pallida (Hornsch.) Aongstr.

Crossomitrium patrisiae (Brid.) C. Muell.

Cyclodictyon varians (Sull.) O. Kuntze

Lepidopilum scabrisetum (Schwaegr.) Steere

Thamniopsis stenodictyon (Sehnem)

Oliveira-e-Silva \& O. Yano

T. incurva (Hornsch.) Buck

T. langsdorffii (Hook.) Buck

LEUCOMIACEAE

Leucomium strumosum (Hornsch.) Mitt.

HYPOPTERYGIACEAE

Hypopterygium tamarisci (Sw.) C. Muell.

Lopidium concinnum (Hook.) Wils.

POTTIACEAE

Barbula indica (Hook.) Spreng.

Chenia leptophylla (C. Muell.) Zand.

Hyophila involuta (Hook.) Jaeg.

Plaubelia sprengelii (Schwaegr.) Zand.

Tortella humilis (Hedw.) Jenn.

CALYMPERACEAE

Calymperes afzelii $\mathrm{Sw}$.

C. erosum C. Muell.

C. lonchophyllum Schwaegr.

C. palisotii Schwaegr. ssp. Richardii

(C. Muell.) S. Edwards

Syrrhopodon gaudichaudii Mont.

S. incompletus Schwaegr.

S. lycopodioides (Brid.) C. Muell.

Syrrhopodon parasiticus (Brid.) Besch.

S. prolifer Schwaegr. var. prolifer

S. prolifer var. papillosus (C. Muell.) Reese

S. rigidus Hook. \& Grev.

DICRANACEAE

Bryohumbertia filifolia (Hornsch.) J. P. Frahm

Campylopus arctocarpus (Hornsch.) Mitt.

C. cryptopodioides Broth.

C. heterostachys (Hampe) Jaeg.

C. pilifer Brid.

C. savannarum (C. Muell.) Mitt.

C. trachyblepharon (C. Muell.) Mitt.

\begin{tabular}{|c|c|c|c|}
\hline $\mathrm{R}-\mathrm{C}$ & OM-SC & UM-EN & $0-930$ \\
\hline $\mathrm{R}-\mathrm{C}$ & OM-SC & UM-EN & $20-360$ \\
\hline R-C-E & $\mathrm{OM}$ & UM & $80-360$ \\
\hline C-E-R & OM-SC-SB & UM-EN & $10-480$ \\
\hline C-E-R & OM-SC-SB & UM-EN & $0-480$ \\
\hline C-R & OM-SC & UM & $80-580$ \\
\hline C-E-R & OM-SC & UM & $260-440$ \\
\hline C-E-R & OM-SC & UM & $100-400$ \\
\hline C-E-T-R & OM-SC & UM-SC & $200-940$ \\
\hline $\mathrm{R}-\mathrm{C}$ & $\mathrm{OM}$ & UM & 220 \\
\hline R-T-C & OM-SB & UM-EN & $0-320$ \\
\hline R-C-E-T & OM-SB & UM & $0-420$ \\
\hline F-C-E-T & OM-SC & UM & $80-420$ \\
\hline E-R-C & OM-SC & UM & $100-380$ \\
\hline $\mathrm{R}$ & OM-SC & UM & $20-160$ \\
\hline $\mathrm{C}$ & - & - & 600 \\
\hline T-R-C-E & OM-SC & UM-EN & $140-420$ \\
\hline R-C-F & OM-SC & UM & $120-260$ \\
\hline R-C-E & $\mathrm{OM}$ & UM & $0-240$ \\
\hline R-C-E & $\mathrm{OM}$ & UM-EN & $0-870$ \\
\hline- & - & - & - \\
\hline $\mathrm{T}$ & $\mathrm{OM}$ & - & 50 \\
\hline $\mathrm{R}$ & - & UM & 0 \\
\hline R-E & OM-SC-SB & UM-EN & $0-380$ \\
\hline $\mathrm{R}$ & $\mathrm{OM}$ & UM & 0 \\
\hline R-C & $\mathrm{SC}$ & - & $0-180$ \\
\hline R-C-E & OM-SC & UM-EN & $0-430$ \\
\hline R-C-E & OM-SC & UM-EN & $0-280$ \\
\hline C-R & $\mathrm{OM}$ & UM & $330-400$ \\
\hline $\mathrm{R}-\mathrm{C}$ & OM-SC & UM & $0-260$ \\
\hline C & - & - & 50 \\
\hline $\mathrm{R}-\mathrm{C}$ & OM-SC & UM & $0-420$ \\
\hline $\mathrm{C}$ & - & - & 600 \\
\hline $\mathrm{E}$ & $\mathrm{SC}$ & UM & 320 \\
\hline R-C-E & OM-SC & UM & $40-580$ \\
\hline R-C-E & OM-SC & UM-EN & $0-350$ \\
\hline C-E-R & $\mathrm{OM}$ & UM-EN & $0-380$ \\
\hline E-R & OM & UM-EN & $0-400$ \\
\hline R-T & $\mathrm{OM}$ & - & $210-250$ \\
\hline $\mathrm{R}$ & OM-SC & $\mathrm{EN}$ & 7400 \\
\hline R-E & OM-SC & UM-EN & $50-240$ \\
\hline $\mathrm{T}$ & $\mathrm{SC}$ & $\mathrm{EN}$ & $0-280$ \\
\hline $\mathrm{R}-\mathrm{T}-\mathrm{C}$ & $\mathrm{OM}$ & UM-EN & $0-320$ \\
\hline $\mathrm{R}-\mathrm{T}^{*}$ & OM-SC & $\mathrm{EN}$ & 0 e 1110 \\
\hline
\end{tabular}

TROPICAL BRYOLOGY 22 (2002) 
Dicranella hilariana (Mont.) Mitt.

Holomitrium arboreum Mitt.

H. crispulum Mart.

H. olfersianum Hornsch.

Leucoloma cruegerianum (C. Muell.) Jaeg.

L. serrulatum Brid.

BRUCHIACEAE

Trematodon longicollis Michx.

LEUCOBRYACEAE

Leucobryum albicans (Schwaegr.) Lindb.

L. clavatum Hampe

L. crispum C. Muell.

L. martianum (Hornsch.) Hampe

Octoblepharum albidum Hedw.

O. cocuiense Mitt.

FISSIDENTACEAE

Fissidens asplenioides Hedw.

F. diplodus Mitt.

F. elegans Brid.

Fissidens guianensis Mont.

$F$. intramarginatus (Hampe) Mitt.

F. longifalcatus C. Muell.

F. mollis Mitt.

$F$. prionodes Mont. fo. hornschuchii

(Mont.) Fleisch.

F. scariosus Mitt.

F. zollingeri Mont.

$\begin{array}{llll}\text { R-T } & \text { OM } & \text { UM } & 110-250 \\ \text { R-C-E } & \text { OM-SC } & \text { UM-EN } & 20-100 \\ \text { R } & \text { OM } & - & 200 \\ \text { R-T } & \text { SC } & \text { UM } & 300 \\ \text { R-E-O } & \text { OM-SC } & \text { UM-EN } & 200-1110 \\ \text { R-C-E } & \text { OM-SC } & \text { UM-EN } & 0-900 \\ & & & \\ \text { T } & \text { OM } & \text { EN } & 40-300 \\ & & & \\ \text { R-C-T } & \text { OM } & \text { UM } & 400-600 \\ \text { C-E-R } & \text { OM } & \text { UM-EN } & 300-950 \\ \text { R-C } & \text { OM-SC } & \text { UM } & 10-650 \\ \text { C-E } & \text { OM } & \text { UM } & 330-600 \\ \text { C-R-T-E } & \text { OM-SC } & \text { UM-EN } & 0-400 \\ \text { R-C } & \text { OM } & \text { UM } & 0 \\ & & & \\ \text { R } & \text { OM-SB } & \text { UM-EN } & 0-220 \\ \text { C-T* } & \text { OM-SC } & \text { UM-EN } & 0-350 \\ \text { C } & - & - & 50 \\ \text { T-R-C } & \text { OM } & \text { UM } & 0-300 \\ \text { T-E } & \text { OM-SC } & \text { UM-EN } & 20-40 \\ \text { R } & \text { OM-SC } & \text { UM-EN } & 320-6100 \\ \text { R-T } & \text { OM } & \text { UM } & 10-20 \\ \text { T-C } & \text { SC } & \text { UM } & 120 \\ \text { E } & \text { OM } & \text { EN } & 440 \\ \text { T-C-R } & \text { OM } & \text { UM } & 450-460 \\ & & & \end{array}$

45\% dos musgos (Fig. 7 - B) são indiferentes quanto ao tipo de ambiente. Segundo Schofield (1985) mesmo quando a precipitação está baixa as briófitas retiram umidade do ar, além de que a própria organização das leivas impede a perda de água para o solo. Almeida Sá (1995) encontrou maior riqueza específica em locais de elevada umidade.

Dumortiera hirsuta, Monoclea gottschei ssp. elongata, Phaeoceros laevis, Plagiochila montagnei, P. kroneana, P. rutilans, Symphyogyna brasiliensis, Callicostella merkelii, C. pallida, Fissidens asplenioides, Helicodontium capillare, Hyophila involuta, Neckeropsis disticha, N. undulata, Philonotis uncinata e Sematophyllum subpinnatum são encontradas submersas em água doce (Tab. 3), embora não sejam briófitas exclusivamente aquáticas. Desses, os gêneros Fissidens, Sematophyllum e Callicostella já foram citados como eventualmente submersos por Yano (1981) e Almeida Sá (1995).
Gradstein \& Pócs (1989) dividem a floresta tropical úmida em duas zonas luminosas. A eufótica mais exposta à irradiação e à dessecação e a oligofótica menos exposta e mais úmida. $\mathrm{Na}$ primeira, são encontradas espécies dos gêneros Frullania e Macromitrium, mais resistentes à seca. Nas áreas estudadas os gêneros acima citados foram coletados sobre rochas totalmente expostas á luz solar e extremamente secas. Características morfológicas como as células superiores mamilosas e basais com pontuações dos filídios de algumas espécies de Macromitrium, as células alongadas e as paredes espessadas de algumas espécies de Frullania, são adaptações a ambientes secos (Proctor 1979).

\section{6- Altitude}

Nas matas tropicais úmidas o aumento do número de indivíduos e a riqueza específica de briófitas é diretamente proporcional à elevação de altitude segundo Schofield (1985), Frahm \& Gradstein (1991), Gradstein (1995) e Gradstein \& Pócs 
A

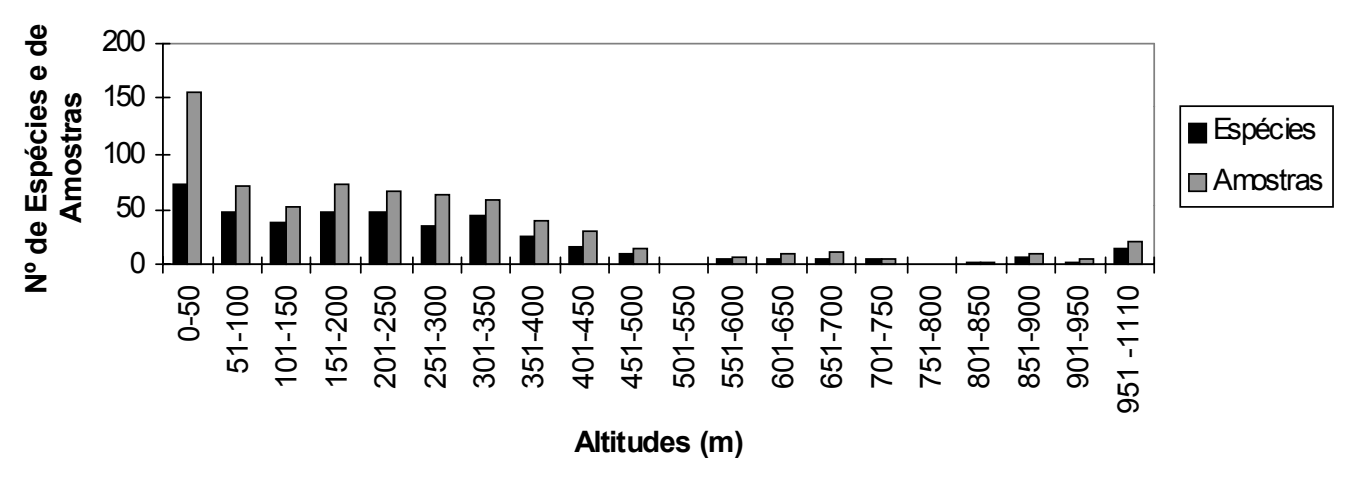

B

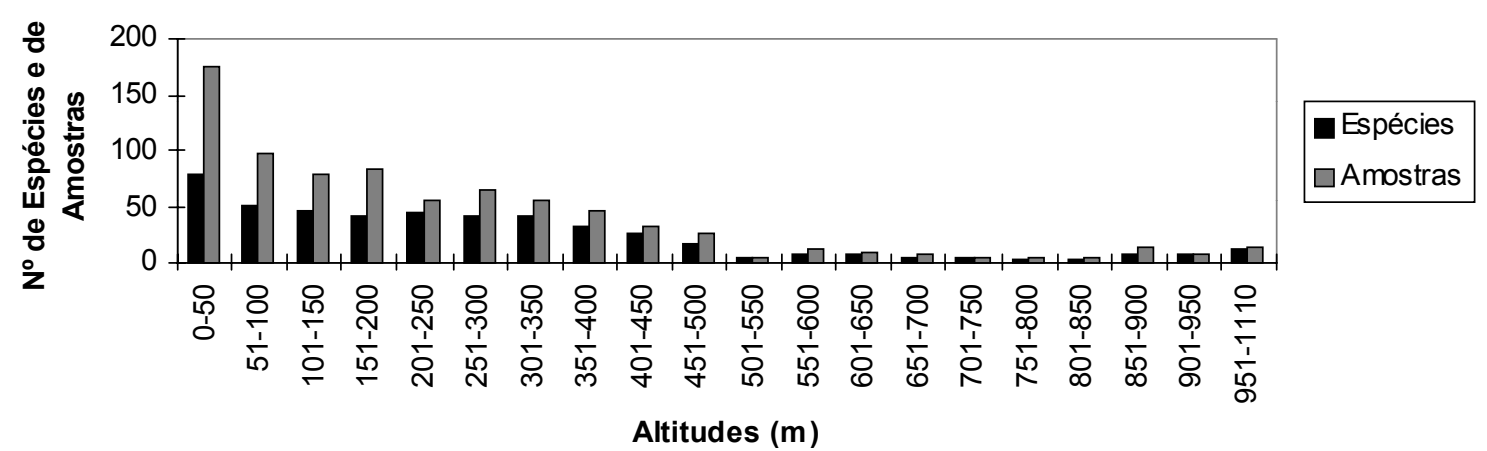

Fig. 8. Distribuição das espécies nos diferentes níveis de altitude encontradas nas áreas estudadas. A). Antóceros e hepáticas. B) Musgos.

(1989). Nas duas áreas estudadas o maior número de indivíduos e de espécies de briófitas cresce entre $0-50 \mathrm{~m}$ de altitude (Fig. 8 e Tab. 3).

As espécies com maior amplitude altitudinal (Tab. 3) são Bryopteris diffusa, B. filicina, Diplasiolejeunea brunnea, Lejeunea flava, $L$. glaucescens, L. minutiloba, Frullania beyrichiana, F. brasiliensis, F. neesii, Plagiochila hypnoides e P. rutilans entre as hepáticas e Hypopterygium tamarisci, Leucoloma cruegerianum, L. serrulatum, Macrocoma frigidum, Macromitrium pellucidum, $M$. punctatum, Pilotrichella flexilis, Orthostichella. pentasticha, Phyllogonium viride, Schlotheimia rugifolia, Sematophyllum subpinnatum, S. sericifolium, Squamidium leucotrichum e $S$. nigricans entre os musgos. Delgadillo et al. (1995) observou o mesmo em relação às espécies de musgos.

As espécies que ocorrem exclusivamente entre 0-50m de altitude (Tab. 3) foram coletadas acima deste nível por outros pesquisadores (Gradstein 1995 e Delgadillo et al. 1995) demonstrando que não são exclusivas dessa faixa altitudinal. Marchantia chenopoda, Campylopus trachyblepharon e Polytrichum juniperinum ocorrem unicamente nos dois extremos, entre 0 $50 \mathrm{~m}$ e acima de $950 \mathrm{~m}$ de altitude (Tab. 3). Delgadillo et al. (1995) cita, Polytrichum juniperinum, como uma espécie que cresce somente em grandes elevações (1130-4700m). Segundo Yano (comunicação pessoal) esta 
espécie como também outras espécies da família Polytrichaceae ocorrem em regiões acima de $800 \mathrm{~m}$ de altitude até $2400 \mathrm{~m}$.

Fig. 8. Distribuição das espécies nos diferentes níveis de altitude encontradas nas áreas estudadas. A). Antóceros e hepáticas. B) Musgos.

\section{Conclusões}

Das famílias encontradas as que apresentam um maior número de espécies são Lejeuneaceae (38), Frullaniaceae e Dicranaceae (13), Calymperaceae (11), Orthotrichaceae e Fissidentaceae (10) e Plagiochilaceae e Meteoriaceae (9).

Dos 231 táxons inventariados nas duas áreas, as espécies Plagiochila rutilans, Sematophyllum subpinnatum, Vesicularia vesicularis e Isopterygium tenerifolium são as mais abundantes. As espécies Vesicularia vesicularis, Plagiochila rutilans, Lejeunea glaucescens, Sematophyllum subpinnatum, Neckeropsis disticha, Helicodontium capillare e Isopterygium tenerifolium são as mais abundantes na RERP, no município de Mangaratiba. As espécies Octoblepharum albidum, Plagiochila hypnoides, $P$. rutilans, Sematophyllum subpinnatum e Lejeunea glaucescens são as mais abundantes na Ilha Grande, município de Angra dos Reis.

A maioria das briófitas é saxícola, ombrófila e prefere ambientes úmidos. Entretanto essas características não são limitantes para o crescimento das mesmas já que adaptações morfológicas ocorrem permitindo a colonização de ambientes hostis. A freqüência absoluta é diretamente proporcional a variabilidade do substrato, demonstrando que não existe uma especificidade espécie-substrato. Os representantes de Anthocerotophyta e Hepatophyta são mais seletivos quanto ao substrato do que os de Bryophyta, visto que a maioria cresce preferencialmente em um só tipo de substrato. Espécies que crescem exclusivamente sobre barrancos e solos apresentam uma freqüência absoluta muito rara. Espécies epífilas são na maioria Hepatophyta, Jungermanniales, principalmente, Lejeuneaceae.
Bryum densifolium, Campylopus trachyblepharon e Fissidens diplodus são espécies com capacidade de suportar ambiente secos das restingas e praias. Dumortiera hirsuta, Monoclea gottschei ssp. elongata, Plagiochila kroneana, P. rutilans, Symphyogyna brasiliensis, Callicostella pallida, Fissidens asplenioides, Helicodontium capillare, Neckeropsis disticha, Philonotis uncinata e Sematophyllum caespitosum, são briófitas que podem crescer, por um curto período de tempo, submersas em água doce.

O índice de similaridade entre as áreas estudadas (69\%), é alto, considerando-se que o índice encontrado entre as duas áreas e outras áreas de Mata Atlântica do estado, Nova Friburgo e a Serra do Itatiaia, foi de $31 \%$ e $9,45 \%$, respectivamente. Das áreas de estudo, a trilha que leva ao Pico do Papagaio na Ilha Grande, e que apresenta as maiores altitudes e a menor influência de atividade antrópica, mostrou a maior diversidade específica.

\section{Agradecimentos}

Agradecemos a todos que contribuíram para a realização dos trabalhos de campo. Na RERP contouse com o apoio do Grupo Mediterrannée, que permitiu o uso do alojamento da reserva, a coleta de material botânico, além de ceder um guia. Em relação à Ilha Grande, pudemos contar até o momento com o auxílio inestimável do Prof. Rogério R. Oliveira da FEEMA e do Dr. Luiz Carlos Sérvulo de Aquino, Diretor de Conservação da Natureza do IEF, que permitiam o uso do alojamento das respectivas Instituições, situados nas áreas correspondentes, além de permitirem a coleta de material. Com a implosão do Presídio Estadual, que ficava situado na Vila Dois Rios, Ilha Grande, contamos com a sede da Universidade do Estado do Rio de Janeiro (UERJ) que recebeu do Estado por comodato (50 anos) as antigas instalações administrativas do complexo presidiário para construção de laboratórios de pesquisa e alojamentos.

\section{Referências bibliográficas}

Alcayaga, E.L. 1985. Bases estatísticas para estudos biológicos. Apostila do Curso de Extensão Departamento de Ciências das Faculdades Integradas de Santa Cruz do Sul, RS. 100p.

Almeida Sá, P.S.A. 1995. Aspectos Florísticos e Ecológicos das Briófitas do Riacho Coité, 
Timbaú-Pe. Dissertação de Mestrado, Universidade Federal de Pernambuco. Recife. 59p.

Barkman, J.J. 1958. Phytosociology and ecology of cryptpgamic epiphytes including a taxonomic survey and description of their vegetation units in Europe. NetherlandVasn Goreum \& Comp. 628 p.

Behar, L.; Yano, O . \& Vallandro, G.C. 1992. Briófitas da Restinga de Setiba, Guaraparí, Espírito Santo. Boletim do Museu de Biologia Mello Leitão 1 : 25-38.

Costa, D.P. 1992. Hepáticas do Pico da Caledônia. Nova Friburgo, Rio de Janeiro, Brasil. Acta Botanica Brasilica 6 (1): 3-39.

Costa, D.P. 1994. Musgos do Pico da Caledônia, município de Nova Friburgo, Estado do Rio de Janeiro, Brasil. Acta Botanica Brasilica 8 (2): 141-191

Dajoz, R. 1983. Ecologia Geral. 41 ed. Petrópolis, ed. Vozes $472 \mathrm{p}$.

Delgadillo, M.C.; Bello, B. \& Cárdenas, M.A.1995. Latmoss. A Catalogue of Neotropical Mosses. Monographs in Systematic Botany - Missouri Botanical Garden 56: 1-191.

Dusén, P. 1903. Sur la flore de la Serra do Itatiaya au Brésil. Arquivos Museu Nacional do Rio de Janeiro 13: 109-119.

Dusén, P. 1909. Beiträge zur flora des Itatiaia. Arkiv för botanik, Uppsala, Stockholm 9 (5): 150.

Frahm, J-P. \& Gradstein, S.R. 1991. An altitudinal zonation of tropical rain forests using bryophytes. Journal of Biogeography 18: 669-678.

Germano, S.R. \& Pôrto, K.C. 1996. Floristic survey of epixylic bryophytes of an area remnant of the Atlantic Forest (Timbaúba - PE, Brazil). 1 - Hepaticopsida (except Lejeuneaceae and Bryopsida. Tropical Bryology 12: $21-28$.

Giminghan, C.H. \& Birse, E.M. 1957. Ecological studies on growth form in bryophytes I. Correlation between growth form and habitat. Journal of Ecology 45 :533-545.

Gradstein, S.R. 1992. The vanishing tropical rain forest as na environment for bryophytes and lichens. in Bates, J.W. \& Farmes, A. M. (eds) Bryophytes and Lichens in a Changing Enviroment, Clarendon Press, Oxford, Chapter $9: 235-257$.

Gradstein, S.R. 1995. Diversity of Hepaticae and Anthocerotae in montane forests of the tropical Andes in Churchil, S.P et al. (eds). Biodiversity and Conservation of
Neotropical Montane Forests New York Botanical Garden pp. 321 - 334.

Gradstein, S.R. \& Pócs, T. 1989. Bryophytes. in Lieth, H. \& Werger, M.A.J. (eds) Tropical Rain Forest Ecosystems, Elsevier Science Publishers B.V., Amsderdam, Chapter 16 :311-325.

Koponen, T. 1978. On the taxonomic value of habitat ecology in mosses in Geissler, P. \& Greene, S.W. (eds). Bryophyte Taxonomy. pp.101105.

Lisboa, R.C.L. 1976. Estudos sobre as vegetações das campinas amazônicas. V. Brioecologia de uma campina amazônica. Acta Amazonica 6 (2): 171-191.

Lisboa, R.C.L. 1985. Avaliação da brioflora de uma área de floresta de terra firme II. Hepaticae. Boletim do Museu paraense Emílio Goeldi, sér. bot. 2 (1): 99-114.

Lisboa, R.C.L. \& Maciel, V.M. 1994. Musgos da Ilha de Marajó I. AFUÁ (Pará). Boletim do Museu paraense Emílio Goeldi, sér. bot. 10 (1): 43-55.

Milliken, W. \& Ratter, J.A. 1989. The Vegetation of the Ilha de Maracá. Edinburgh Royal Botanical Garden 10,277p.

Oliveira-e-Silva, M.I.M.N. \& Yano, O. 1998. Ocorrências novas de briófitas para o Brasil. Revista Brasileira de Botanica 21 (2) :125134

Pócs, T. 1982. The Tropical Bryophytes. in Smith, A.J.E. Bryophytes Ecology, London, Chapman and Hall, p. 54-104.

Pôrto, K.C. 1992. Bryoflores d'une fôret de plaine et d'une fôret d'altitude moyenne dans l'Etat de Pernambuco (Brésil): 2. Analyse écologique comparative des fôrets. Cryptogamie, Bryol. Lichénol. 13 (3): 187 219.

Proctor, M.C.F. 1979. Structure and ecophysiological adaptation in bryophytes in Clarke, G.C.S. \& Duckett, J.G. (eds) : 479509, Academic Press, London and New York.

Richards, P.W. 1984. The Ecology of Tropical forest bryophytes. in Schuster, R. M. (ed.) New Manual of Bryology. J. Hattori Bot. Lab. 2: 1232-1270.

Sehnem, A. 1969. Musgos Sul-brasileiros 1, Pesquisas, sér. bot. 27: 1-41.

Sehnem, A. 1970. Musgos Sul-brasileiros 2, Pesquisas, sér. bot. 28: 1-117.

Sehnem, A. 1972. Musgos Sul-brasileiros 3, Pesquisas, sér. bot. 29: 1-70.

Sehnem, A. 1976. Musgos Sul-brasileiros 4, 


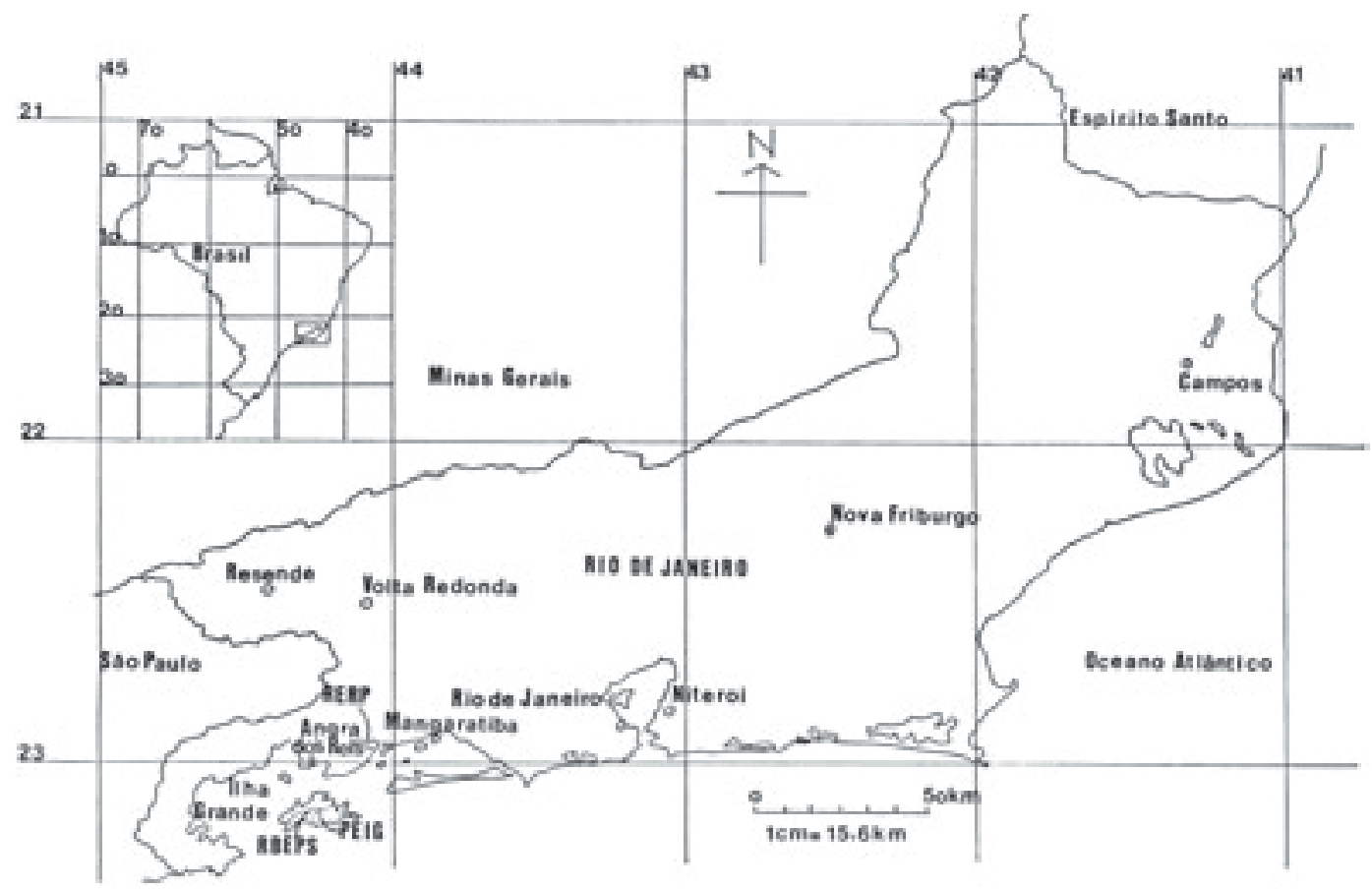

Fig. 1: Mapa da localização das áreas de estudo.

Pesquisas, sér. bot. 30: 1-36.

Sehnem, A. 1978. Musgos Sul-brasileiros 5, Pesquisas, sér. bot. 32: 1-170.

Sehnem, A. 1979. Musgos Sul-brasileiros 6, Pesquisas, sér. bot. 33: 1-149.

Sehnem, A. 1980. Musgos Sul-brasileiros 7, Pesquisas, sér. bot. 34: 1-121.

Schäfer-Verwimp, A. 1991. Contribution to the knowledge of the bryophyte flora of Espírito Santo, Brasil. J. Hattori Bot. Lab. 69: 147170.

Schofield, W.B. 1985. Introduction to Bryology. Macmillan Publ. Co., New York.

Sim, T.R. 1926. The Bryophyta of South Africa. Transactions Royal Society of South Africa 15: $1-475$.

Slack, N.G. 1977. Species diversity and community structure in bryophytes: New York State studies. Bulletin of New York State Museum 428: 1-70.

Valentin, J.L. 1995. Agrupamento e Ordenação in Peres-Neto, P.R.; Valentin, J.L. \& Fernandez, F.A.S. (eds.). Oecologia Brasiliensis - Tópicos em Tratamento de Dados Biológicos, Programa de Pós-
Graduação em Ecologia - Instituto de Biologia - UFRJ, Rio de Janeiro - RJ. pp.2755.

Vital, D.M.; Giancotti, C. \& Pursell, R.A. 1991. The bryoflora of Fernando de Noronha, Brasil. Tropical Bryology 4:23-24.

Yano, O. 1981. Bryophyta in Hulbert, S.H.; Rodrigues, G. \& Santos, N.D. (eds). Aquatic Biota of Tropical South America, Part. 2: Anarthropa. San Diego State University, San Diego, California, XI + 298 pp.

Yano, O. 1990. Musgos do Parque Estadual da Ilha do Cardoso, Estado de São Paulo: Sphagnaceae, Rhizogoniaceae, Mniaceae, Racopilaceae e Phyllogoniaceae. Anais do II Simpósio de Ecossistemas, Águas de Lindóia, S.P. 1: 411-438.

Yano, O. 1992a. Briófitas da Ilha de Maracá, Roraima, Brasil. Acta Amazonica 22 (4): 535-539.

Yano, O. 1992b. Leucobryaceae (Bryopsida) do Brasil. Tese de Doutorado. São Paulo. Universidade de São Paulo. 318 pp.

Yano, O. \& Costa, D.P. 1993. Briófitas da Restinga da Massambaba, Rio de Janeiro. Anais do III Simpósio de Ecossistemas da Costa Brasileira, ACIESP, Serra Negra, 3:144-157. 
de Oliveira e Silva et al.

TROPICAL BRYOLOGY 22 (2002) 